



\section{Annual Report Fiscal Year 2008}

Ernest Orlando Lawrence Berkeley National Laboratory University of California Berkeley

Berkeley, California

January 2009

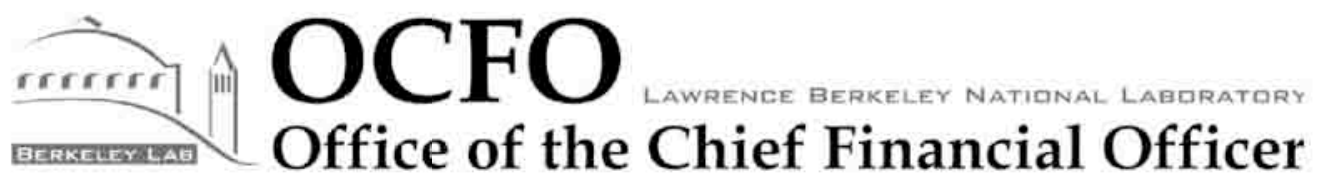




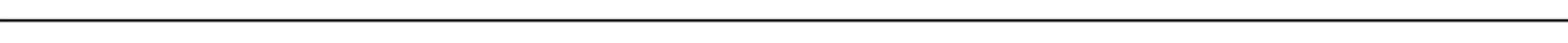




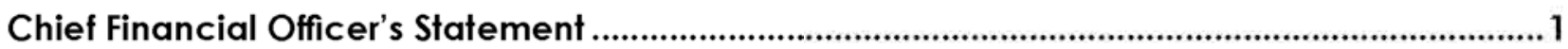

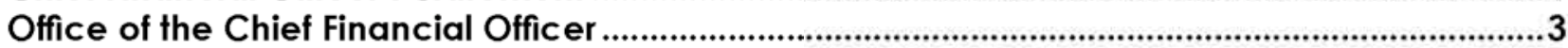

Lawrence Berkeley National Laboratory, University of California ..................................................4

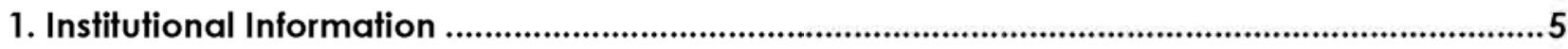

Where Did Your Program Dollars Go in FY2008? | Figure 1.1 ...............................................6

Cost Trend by Expense Category, FY2004 - FY2008 (\$M and \% of Total) Table 1.1 .............7

Cost By Direct Funding Source by Division, FY2004 - FY2008 (\$K) | Table 1.2 ....................8

Cost by Direct Funding Source by Division, FY2008 (\$K) | Table 1.2a ................................9

Cost By Direct Funding Source by Division, FY2007 (\$K) | Table 1.2b ..................................10

Cost By Direct Funding Source by Division, FY2006 (\$K) | Table 1.2c.................................11

Cost By Direct Funding Source by Division, FY2005 (\$K) | Table 1.2d.................................12

Cost By Direct Funding Source by Division, FY2004 (\$K) | Table 1.2e.................................13

Indirect Budget Costs by Division, FY2008 (\$K) | Table 1.3 .................................................14

Average FTE Breakdown by Division, FY2008 (\$K) | Table 1.4 ..............................................15

Funds Held for Others' Costs, FY2004 - FY2008 (\$K) | Table 1.5 .........................................16

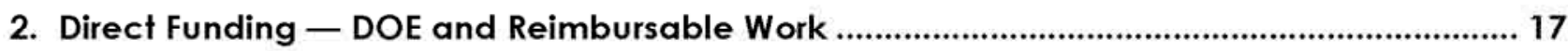

LBNL Fund Trends (BA) by Funding Source (\$K) | Table 2.1 ...............................................20

LBNL Cost Trends by Funding Source (\$K) | Table 2.2 ......................................................22

Laboratory Funding and Costs by Source (\$K) | Table 2.3...............................................24

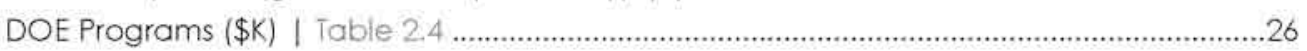

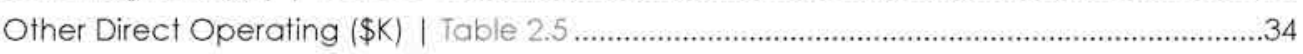

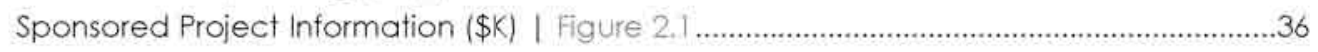

3. Indirect Budgets

Indirect Budgets — FY2008 Costs (\$M) | Figure 3.1 …............................................................38

Institutional Overhead Costs as a Percent of Operating Costs,

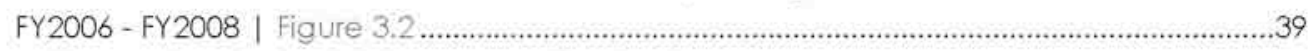

Institutional Costs by Division, FY2008 (\$K) | Table 3.1 .......................................................40

Institutional FTEs Charged by Division, FY2008 | Table 3.2 …...............................................41

Payroll Burden Summary (\$M) | Figure 3.3 .................................................................. 42

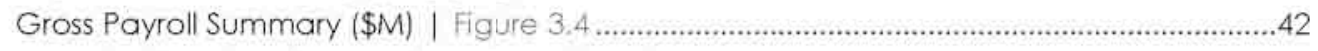

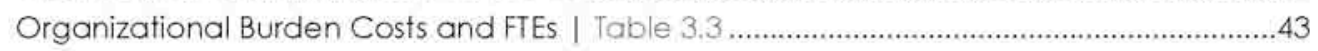

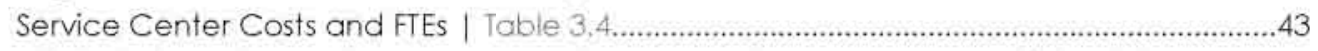

Distributed Recharges by Resource Category Trends, FY2004 - FY2008 (\$K) Table 3.5 .......44 
Balance Sheet Comparative Statement of Financial

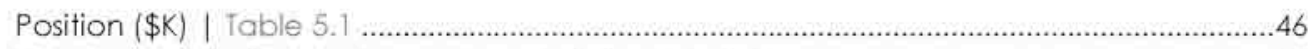

Summary of Significant Accounting Policies | Note T ......................................................4 47

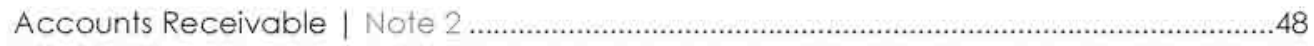

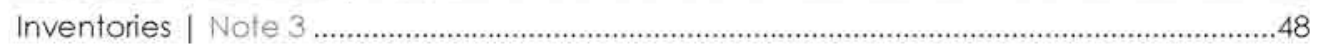

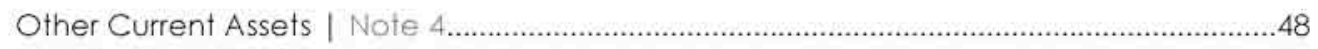

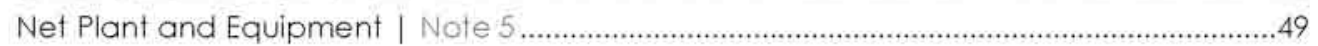

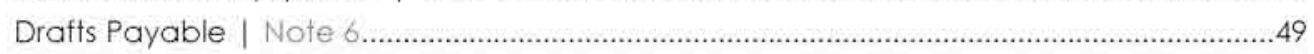

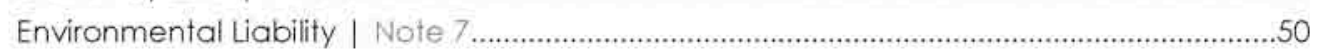

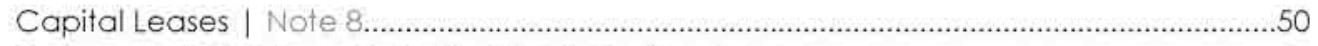

Environment, Safety and Health (ES\&H) Liability | Note 9 ................................................50

5. Procurement and Property Management........................................................................... 51

Requisitions Submitted by Laboratory Divisions | Table 5.1 52

Purchases Placed Using Purchase Orders/Subcontracts | Table 5.2 ….............................53

Purchases Placed Using P-Card | Table 5.3 .......................................................................53

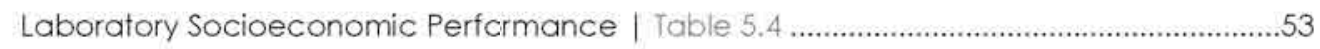

Property Management Activity | Table 5.5 .........................................................................54

6. Data from Other DOE Laboratories …....................................................................................... 55

Other Laboratories for Which Financial Information is Available | Table 6.1 ......................57

Summary Cost Data for DOE Laboratories, FY2004 - FY2007 (\$M) | Table 6.2 ....................58

Overhead Information for DOE Laboratories, FY2007 | Table 6.3 …...................................59

Overhead Costs as a Percentage of Operating Costs

for DOE Laboratories, FY2004 - FY2007 | Table 6.4 …..........................................................60

Functional Support Costs as a Percent of Total Costs, FY2002 - FY2007 | Figure 6.1 ...........60

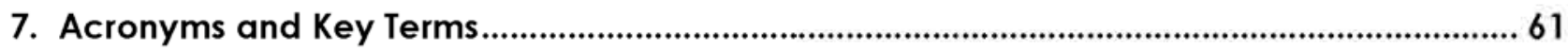




\section{Chief Financial Officer's Statement}

It is with great pleasure that I present to you the 2008 Chief Financial Officer's Annual Report. The data included in this report has been compiled from the Budget Office, The Controller, Procurement and Property Management and the Sponsored Projects Office. Also included are some financial comparisons with other DOE Laboratories and a glossary of commonly used acronyms. I hope that you will find it useful as a reference tool.

$\ln$ 2008, the Office of the Chief Financial Officer (OCFO) senior management team convened to update its Strategic Plan taking into consideration the exciting new science being performed at the Laboratory that requires innovative new financial models. We took pride in the accomplishments from our original plan, and look forward to implementing the ambitious improvements noted in the updated plan. Listed below are some of our more important achievements.

Crucial to the success of any OCFO achievement is first and foremost that employees are safe and that they have all the tools and training they need to perform their work in the safest manner possible. To that end, the OCFO staff produced two safety videos on packing and moving, as a large segment of OCFO staff that have been housed in an off-site facility will be moving back up to "the hill" early in 2009. These videos were such a hit that they were endorsed by Laboratory senior management and used institutionally.

We also made significant efforts in managing the safety of our matrixed staff who work in every division at the Laboratory. To better serve all employees who use financial systems, we incorporated ergo friendly usability design considerations into two new Lab systems, travel and budget.

FY2008 saw the addition of six new commodities to our eBuy procurement program. This program has saved the Laboratory over $\$ 15 \mathrm{M}$ to date and we are on schedule to save the $\$ 30 \mathrm{M}$ required by our Contract. The Procurement Department also implemented a professional training plan resulting in reduced selfassessment findings, increased customer satisfaction, and improved cycle times.

The OCFO collaborated with Information Technology Division partners to develop a financial systems IT strategy that improves awareness and understanding of systems capabilities. Strengthening the bond with the IT
Division has enabled us to deliver better decision support and will make the implementation of new systems run more smoothly from the customer perspective. We look forward to working much closer with IT in 2009 continually looking for ways to improve our processes and systems.

This past fiscal year saw the OCFO usher in several systems improvements to strengthen the Laboratory's financial and business processes. They include the Travel and Expense System (TREX), Budget and Planning System, Events Database, RegOnline Database, and improvements to Procurement reporting.

TREX is the new system that replaced Gelco for reporting travel expenses providing a user-friendly interface that simplifies data entry and approvals. This system was designed by the people who use it the most, travelers and travel arrangers, and incorporates enhanced internal controls and integration with other Laboratory business systems. Initial response to the new system is very positive and we will continue refining it and adding new features in the future.

Phase two of the new Budget and Planning System, the Planning module, was successfully implemented in the Fall of 2008. This new module enhances the ability to develop and maintain more consistent, accurate spending projections throughout the year. The roll-out of phase two completes the implementation of the Budget and Planning System which now will contain funding and planned spending information.

The OCFO developed a database to track conferences and meetings to ensure that we are in compliance with a new Department of Energy Conference Order. This database allows the Laboratory to track how many attendees travel to various conferences as well as consolidate allinformation necessary for our Conference Services group to provide more efficient and effective service to our customers. The database makes it easier to track documents needed for the approval process. as well as costs and status of each event. 
Also related to conferences, we now have a fully automated online registration and attendee management system for the Laboratory called RegOnline. This system is web-based and delivers credit card processing, one click report generation, event revenue management, and sends automatic confirmation emails. This system has dramatically improved the registration process for the Laboratory. The OCFO delivered training to the entire Lab community on both of these new systems.

Training is a major component of the OCFO's mission. In addition to delivering training on the two systems noted above, this year we also introduced training for funds control and procedures, as well as the proper utilization of food services. In order to maintain compliance with DOE policy, the OCFO provided training for the Laboratory community on the proper use of food service at meetings. This outreach increased awareness and understanding of the policy and improved compliance and cost allowability.

Field Operations managers and staff successfully developed and implemented a web-based funds control training module for scientific and administrative personnel responsible for managing financial resources. Also, a new Laboratory-wide policy on the major financial management roles and responsibilities for all employees with fiduciary responsibilities was developed and implemented.

The Property Management Improvement Project (PMIP), which was completed this year, is a decentralized Laboratory-wide system for property management. It has improved controls, accuracy and accountability of our property management responsibilities, and has given the divisions more control by allowing them to process their own transactions. Extensive training was provided by OCFO for all division property representatives. We now have a clean, reliable database that will allow us to manage our inventories with much more efficiency.

As you can see, FY2008 was a very busy year for the staff of the OCFO. I am extremely proud of their achievements in service to the legendary science performed at Lawrence Berkeley National Laboratory.

Sincerely,

Jeffrey A. Fernandez

Chief Financial Officer 


\section{Office of the Chief Financial Officer}

Organization

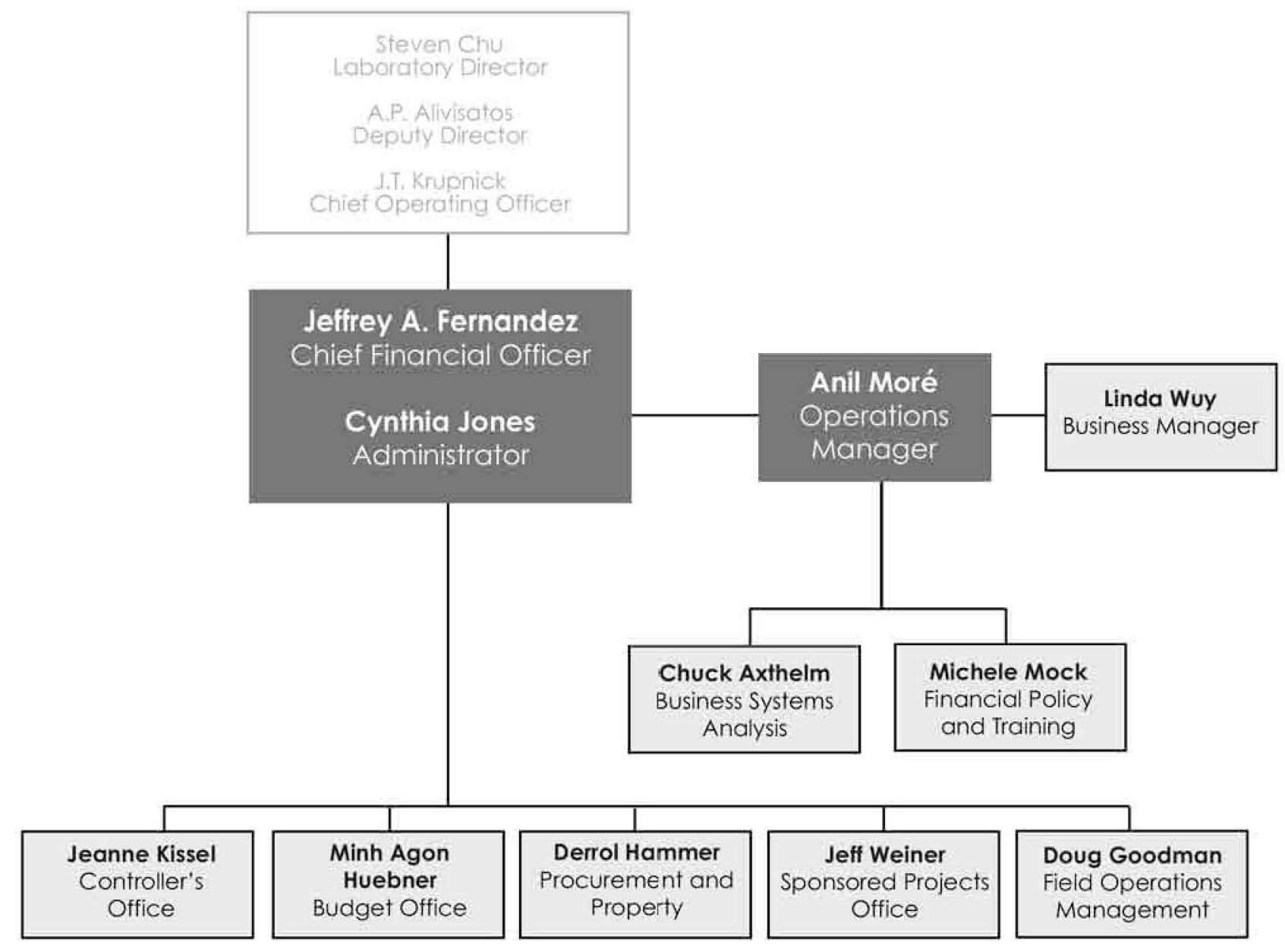




\section{Lawrence Berkeley National Laboratory, University of California}

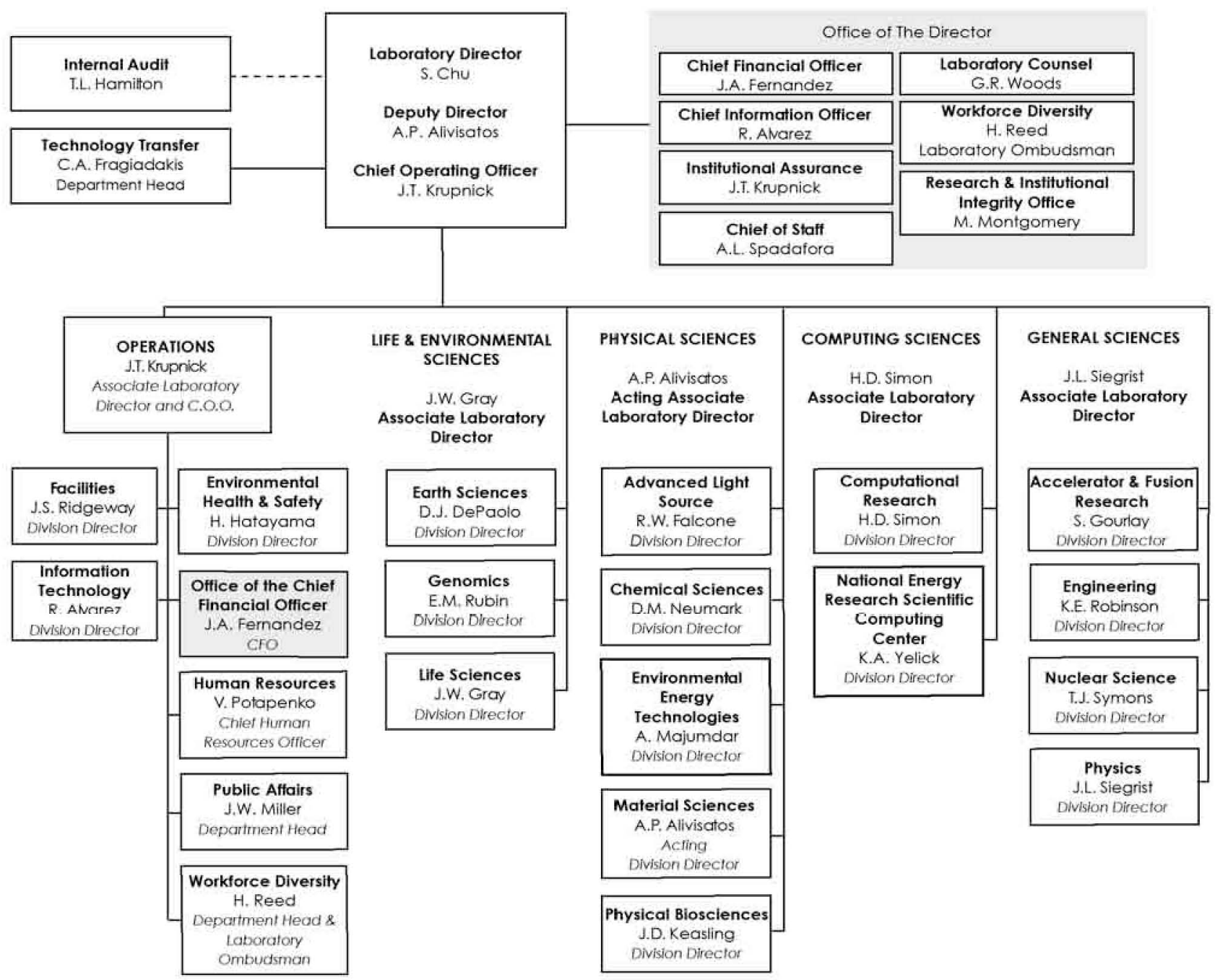


1. Institutional Information 
Figure 1.1

\section{Where Did Your Program Dollars Go in FY2008?}

\begin{tabular}{|c|c|c|c|c|}
\hline \multirow[b]{2}{*}{ Expenses } & \multicolumn{4}{|c|}{ LBNL Cost Breakdown per Dollar } \\
\hline & DOE Operating Costs & $\begin{array}{l}\text { DOE Integrated } \\
\text { Contractors Costs }\end{array}$ & $\begin{array}{c}\text { Construction and } \\
\text { Equipment }\end{array}$ & WFO NON-DOE \\
\hline \multicolumn{5}{|l|}{ DIRECT: } \\
\hline \multicolumn{5}{|l|}{ Direct Labor: } \\
\hline UC Labor (a) & $\$ 0.35$ & $\$ 0.32$ & $\$ 0.12$ & $\$ 0.37$ \\
\hline Contract Labor & $\$ 0.00$ & $(\$ 0.00)$ & $\$ 0.00$ & $\$ 0.00$ \\
\hline Org. Burden (b) & $\$ 0.05$ & $\$ 0.06$ & $\$ 0.02$ & $\$ 0.07$ \\
\hline Subtotal Direct Labor & $\$ 0.40$ & $\$ 0.38$ & $\$ 0.14$ & $\$ 0.44$ \\
\hline \multicolumn{5}{|l|}{ Other Direct: } \\
\hline Services & $\$ 0.22$ & $\$ 0.01$ & $\$ 0.20$ & $\$ 0.14$ \\
\hline Materials & $\$ 0.09$ & $\$ 0.07$ & $\$ 0.56$ & $\$ 0.07$ \\
\hline Utilities & $\$ 0.02$ & $\$ 0.00$ & $\$ 0.00$ & $\$ 0.01$ \\
\hline Other Expenses (c) & $\$ 0.00$ & $\$ 0.01$ & $\$ 0.00$ & $\$ 0.01$ \\
\hline Recharges $(b, d)$ & $\$ 0.00$ & $\$ 0.29$ & $\$ 0.01$ & $\$ 0.04$ \\
\hline Travel & $\$ 0.02$ & $\$ 0.01$ & $\$ 0.00$ & $\$ 0.02$ \\
\hline Subtotal Other Direct & $\$ 0.35$ & $\$ 0.39$ & $\$ 0.77$ & $\$ 0.28$ \\
\hline Total Direct & $\$ 0.75$ & $\$ 0.76$ & $\$ 0,91$ & $\$ 0,72$ \\
\hline \multicolumn{5}{|l|}{ INDIRECT: } \\
\hline Procurement & $\$ 0.01$ & $\$ 0.01$ & $\$ 0.03$ & $\$ 0.01$ \\
\hline Travel & $\$ 0.00$ & $\$ 0.00$ & $\$ 0.00$ & $\$ 0.00$ \\
\hline G\&A (Other Inst.) & $\$ 0.23$ & $\$ 0.23$ & $\$ 0.06$ & $\$ 0.27$ \\
\hline Total Indirect & $\$ 0.25$ & $\$ 0.24$ & $\$ 0.09$ & $\$ 0.28$ \\
\hline Total Expenses & $\$ 1.00$ & $\$ 1.00$ & $\$ 1.00$ & $\$ 1.00$ \\
\hline \multicolumn{5}{|c|}{$\begin{array}{l}\text { Note: Minor variances may occur due to rounding. } \\
\text { (a) UC Labor includes salary and benefits for Scientists/Engineers, Admin., Students/GSRAs and Campus Labor. } \\
\text { (b) Distributed activities used by direct funded programs. } \\
\text { (c) Includes miscellaneous expenses (stipends, sales tax, freight, etc.). } \\
\text { (d) Includes recharges credited back to direct operating accounts such as ALS and ESnet. }\end{array}$} \\
\hline
\end{tabular}

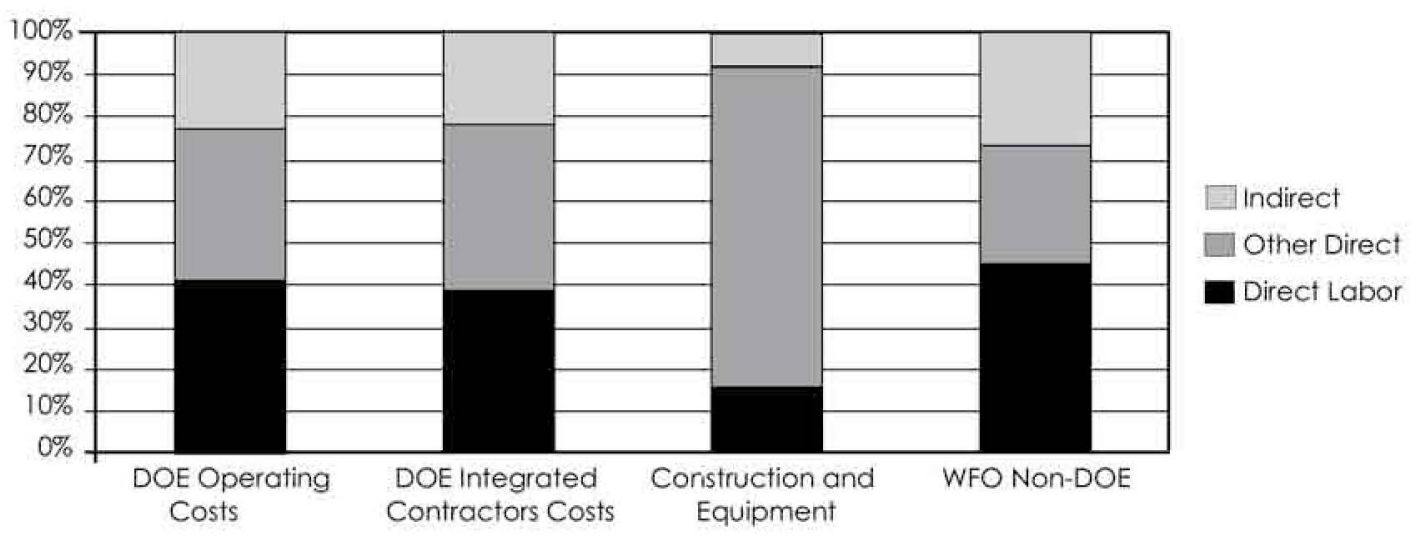


Table 1.1

\section{Cosł Trend by Expense Category, FY2004 - FY2008}

\begin{tabular}{|c|c|c|c|c|c|c|c|c|c|c|}
\hline \multirow{2}{*}{ Expenses } & \multicolumn{2}{|c|}{ FY2004 } & \multicolumn{2}{|c|}{ FY2005 } & \multicolumn{2}{|c|}{ FY2006 } & \multicolumn{2}{|c|}{ FY2007 } & \multicolumn{2}{|c|}{ FY2008 } \\
\hline & $\$ M$ & $\%$ & $\$ M$ & $\%$ & $\$ M$ & $\%$ & $\$ M$ & $\%$ & $\$ M$ & $\%$ \\
\hline \multicolumn{11}{|l|}{ DIRECT: } \\
\hline \multicolumn{11}{|l|}{ Direct Labor: } \\
\hline UC Labor (a) & 178.2 & $35.4 \%$ & 174.8 & $33.4 \%$ & 175.4 & $33.9 \%$ & 180.3 & $34.9 \%$ & 191.7 & $32.5 \%$ \\
\hline Contract Labor & 1.1 & $0.2 \%$ & 0.8 & $0.2 \%$ & 0.9 & $0.2 \%$ & 1.3 & $0.3 \%$ & 1.2 & $0.2 \%$ \\
\hline Org. Burden (b) & 28.7 & $5.7 \%$ & 27.3 & $5.2 \%$ & 29.2 & $5.6 \%$ & 29.8 & $5.8 \%$ & 30,4 & $5.2 \%$ \\
\hline Subtotal Direct Labor & 208.0 & $41.3 \%$ & 202.9 & $38.7 \%$ & 205.6 & $39.7 \%$ & 211.4 & $40.9 \%$ & 223.3 & $37.9 \%$ \\
\hline \multicolumn{11}{|l|}{ Other Direct: } \\
\hline Services & 79.6 & $15.8 \%$ & 102.1 & $19.5 \%$ & 91.7 & $17.7 \%$ & 84.0 & $16.3 \%$ & 117.2 & $19.9 \%$ \\
\hline Materials & 73,9 & $14.7 \%$ & 75.5 & $14.4 \%$ & 68.4 & $13.2 \%$ & 68.0 & $13.2 \%$ & 82.1 & $13.9 \%$ \\
\hline Utilities & 6.0 & $1.2 \%$ & 7.1 & $1.4 \%$ & 5.1 & $1.0 \%$ & 6.2 & $1.2 \%$ & 7.3 & $1.2 \%$ \\
\hline Other Expenses (c) & 1.8 & $0.4 \%$ & 1.1 & $0.2 \%$ & 1.9 & $0.4 \%$ & 2.7 & $0.5 \%$ & 2.8 & $0.5 \%$ \\
\hline Recharges $(b, d)$ & 9.8 & $1.9 \%$ & 8.8 & $1.7 \%$ & 10.6 & $2,0 \%$ & 8.4 & $1.6 \%$ & 8.9 & $1.5 \%$ \\
\hline Travel & 9.4 & $1.9 \%$ & 9.4 & $1.8 \%$ & 9.8 & $1.9 \%$ & 7.9 & $1.5 \%$ & 9.4 & $1.6 \%$ \\
\hline Subtotal Other Direct & 180.5 & $35.8 \%$ & 204.0 & $38.9 \%$ & 187.4 & $36.2 \%$ & 177.2 & $34.3 \%$ & 227.7 & $38.6 \%$ \\
\hline Total Direct & 388.5 & $77.1 \%$ & 406.8 & $77.7 \%$ & 393.0 & $76.0 \%$ & 388.5 & $75.2 \%$ & 451.0 & $76.5 \%$ \\
\hline \multicolumn{11}{|l|}{ INDIRECT: } \\
\hline Procurement & 7.1 & $1.4 \%$ & 6.6 & $1.3 \%$ & 7.5 & $1.5 \%$ & 7.4 & $1.4 \%$ & 8.2 & $1.4 \%$ \\
\hline Travel & 0.9 & $0.2 \%$ & 0.9 & $0.2 \%$ & 0.8 & $0.2 \%$ & 0.9 & $0.2 \%$ & 1.2 & $0.2 \%$ \\
\hline Space (e) & 7.5 & $1.5 \%$ & 8.7 & $1.7 \%$ & 0.0 & $0.0 \%$ & 0.0 & $0.0 \%$ & 0.0 & $0.0 \%$ \\
\hline G\&A (Other inst.) & 99.7 & $19.8 \%$ & 100.7 & $19.2 \%$ & 115.8 & $22.4 \%$ & 119.6 & $23.2 \%$ & 129.2 & $21.9 \%$ \\
\hline Total Indirect & 115.2 & $22.9 \%$ & 116.9 & $22.3 \%$ & 124.2 & $24.0 \%$ & 127.8 & $24.8 \%$ & 138.5 & $23.5 \%$ \\
\hline Total Expenses & 503.7 & $100.0 \%$ & 523.7 & $100.0 \%$ & 517.2 & $100.0 \%$ & 516.4 & $100.0 \%$ & 589.5 & $100.0 \%$ \\
\hline \multicolumn{11}{|c|}{ Note: Minor variances may occur due to rounding. } \\
\hline \multicolumn{11}{|c|}{ (a) UC Labor includes salary and benefits for Scientists/Eng } \\
\hline \multicolumn{11}{|c|}{ (b) Distributed activities used by direct funded programs. } \\
\hline \multicolumn{11}{|c|}{ (c) Includes miscellaneous expenses (stipends, sales tax, freight, etc.). } \\
\hline \multicolumn{11}{|c|}{$\begin{array}{l}\text { (d) Includes recharges credited back to direct operating accou nts such as ALS and ESnet (In FY2004 Annual Report these were } \\
\text { included in the Other Expenses category). }\end{array}$} \\
\hline (e) Space rate eliminated in FYz2006 & its $m$ & d to $G 8$ & Site S & port). & & & & & & \\
\hline
\end{tabular}


Table 1.2

\section{Cost By Direct Funding Source by Division, FY2004 - FY2008 (\$K)}

\begin{tabular}{|c|c|c|c|c|c|}
\hline Division & FY2004 & FY2005 & FY2006 & FY2007 & FY2008 \\
\hline Accelerator \& Fusion Research & 27,375 & 27,163 & 25,595 & 28,099 & 26,042 \\
\hline Advanced Light Source & 43,067 & 45,023 & 44,180 & 48,906 & 51.508 \\
\hline Chemical Sciences & 12,578 & 12,351 & 12,554 & 14,877 & 17,002 \\
\hline Computing Sciences $(a)$ & 0 & 10 & 79,614 & 77,355 & 101,133 \\
\hline Computational Research (a) & 19,767 & 18,828 & $=$ & - & - \\
\hline NERSC Center (a) & 29,470 & 41,299 & - & - & - \\
\hline Information Technology (a) & 26.203 & 28,195 & 3,852 & 3,391 & 3,634 \\
\hline Environmental Energy Technologies & 54,257 & 51.514 & 53,052 & 45,763 & 52,004 \\
\hline Engineering & 4,557 & 4,503 & 5,408 & 8,429 & 13,351 \\
\hline EH\&S & 6,262 & 5,780 & 7,360 & 5,493 & 3,490 \\
\hline Earth Sciences & 29.721 & 28,954 & 31,036 & 29,856 & 31.027 \\
\hline Facilities & 10,050 & 41,275 & 31,492 & 12,244 & 17,076 \\
\hline Genomics & 8.956 & 8,198 & 9,733 & 10,437 & 8,731 \\
\hline Genomics-JGl & 50,136 & 46,706 & 43,105 & 45,461 & 50,839 \\
\hline Life Sciences & 42,084 & 43,113 & 47,788 & 51,929 & 56.872 \\
\hline Materials Sciences & 51,481 & 35,352 & 40,048 & 50,657 & 55,835 \\
\hline Nuclear Science & 21,676 & 28,781 & 26,501 & 28,098 & 26,774 \\
\hline Physical Biosciences & 31.692 & 28,680 & 29.167 & 25,228 & 44.219 \\
\hline Physics & 33.805 & 27,305 & 26.978 & 30,373 & 29,984 \\
\hline Lab Directorate/Other & 664 & 924 & 752 & 730 & 858 \\
\hline Other (b) & 167 & $(116)$ & $(1,060)$ & $(947)$ & (880) \\
\hline Division Total & 503,969 & 523,837 & 517,155 & 516,382 & 589,499 \\
\hline
\end{tabular}

Note: Minor variances may occur due to rounding.

(a) Computational Research, NERSC Center as well as the ESNET portion of Information Technology became part of Computing Sciences in FY2006

(b) Primarily Work for Other charge to offset Safeguards and Security activities. 
Table $1.2 a$

\section{Cost by Direct Funding Source by Division, FY2008 (\$K)}

\begin{tabular}{|c|c|c|c|c|c|c|c|}
\hline \multirow[b]{2}{*}{ Division } & \multicolumn{7}{|c|}{ FY2008 } \\
\hline & $\begin{array}{c}\text { DOE } \\
\text { Operating }\end{array}$ & $\begin{array}{c}\text { DOE Integrated } \\
\text { Confractors } \\
\text { Costs }\end{array}$ & $\begin{array}{c}\text { WFO } \\
\text { Federal }\end{array}$ & $\begin{array}{l}\text { WFO Non- } \\
\text { Federal }\end{array}$ & $\begin{array}{l}\text { Operating } \\
\text { Subtotal }\end{array}$ & $\begin{array}{l}\text { Capital \& } \\
\text { Equipment }\end{array}$ & Total \\
\hline Accelerator \& Fusion Research & 19,426 & 1,514 & 1,441 & 799 & 23,180 & 2,862 & 26,042 \\
\hline Advanced Light Source & 44,552 & 7 & 0 & 865 & 45,424 & 6,084 & 51.508 \\
\hline Chemical Sciences & 14,236 & 75 & 599 & 79 & 14,990 & 2,012 & 17,002 \\
\hline Computing Sciences $(a)$ & 87,106 & 2,395 & $T, 464$ & 1,227 & 92,693 & 8,440 & 101,133 \\
\hline Computational Research (a) & 0 & 0 & 0 & 0 & 0 & 0 & 0 \\
\hline NERSC Center $(a)$ & 0 & 0 & 0 & 0 & 0 & 0 & 0 \\
\hline Information Technology (a) & 2,455 & 0 & 0 & 45 & 2,500 & 1,134 & 3,634 \\
\hline Environmental Energy Technologies & 30,890 & 1.513 & 5,701 & 12,797 & 50,901 & 1.103 & 52,004 \\
\hline Engineering & 2,814 & 213 & 322 & 516 & 3,865 & 9,485 & 13,351 \\
\hline EH\&S & 3,490 & 0 & 0 & 0 & 3,490 & 0 & 3,490 \\
\hline Earth Sciences & 21,996 & 2,171 & 1,339 & 4,681 & 30,186 & 841 & 31.027 \\
\hline Facilities & 4,174 & 0 & 0 & 0 & 4,174 & 12,902 & 17,076 \\
\hline Genomics: & 2,522 & 0 & 6,043 & 34 & 8,599 & 132 & 8,731 \\
\hline Genomics - JGI & 45,068 & 301 & 357 & 945 & 46,671 & 4,168 & 50,839 \\
\hline Life Sciences & 11,452 & 20 & 36,844 & 6.876 & 55.191 & 1,680 & 56,872 \\
\hline Materials Sciences & 42,030 & 123 & 1,004 & 7,238 & 50,396 & 5,439 & 55,835 \\
\hline Nuclear Science & 17,280 & 90 & 2,296 & 3,533 & 23,199 & 3,575 & 26.774 \\
\hline Physical Biosciences & 33.778 & 515 & 6,071 & 3,761 & 44,126 & 93 & 44,219 \\
\hline Physics & 22,481 & 791 & 715 & 353 & 24,341 & 5,644 & 29.984 \\
\hline Laboratory Directorate/Other & 743 & 0 & 0 & 115 & 858 & 0 & 858 \\
\hline Other (b) & $(947)$ & 68 & 0 & 0 & $(880)$ & 0 & (880) \\
\hline Division Total & 405,548 & 10,296 & 64,195 & 43,864 & 523,904 & 65,595 & 589,499 \\
\hline
\end{tabular}


Table $1.2 \mathrm{~b}$

\section{Cost By Direct Funding Source by Division, FY2007 (\$K)}

\begin{tabular}{|c|c|c|c|c|c|c|c|}
\hline \multirow[b]{2}{*}{ Division } & \multicolumn{7}{|c|}{ FY2007 } \\
\hline & $\begin{array}{c}\text { DOE } \\
\text { Operating }\end{array}$ & $\begin{array}{c}\text { DOE Integrated } \\
\text { Contractors } \\
\text { Costs }\end{array}$ & $\begin{array}{c}\text { WFO } \\
\text { Federal }\end{array}$ & $\begin{array}{l}\text { WFO Non- } \\
\text { Federal }\end{array}$ & $\begin{array}{l}\text { Operating } \\
\text { Subtotal }\end{array}$ & $\begin{array}{l}\text { Capital \& } \\
\text { Equipment }\end{array}$ & Total \\
\hline Accelerator \& Fusion Research & 20,818 & 660 & 2,662 & 1,469 & 25.609 & 2,490 & 28,099 \\
\hline Advanced Light Source & 42,453 & 13 & 0 & 809 & 43,275 & 5,632 & 48,906 \\
\hline Chemical Sciences & 12,457 & 0 & 704 & 115 & 13,276 & 1,601 & 14.877 \\
\hline Computing Sciences (a) & 67,615 & 2,902 & 1,376 & 1,202 & 73,096 & 4,259 & 77,355 \\
\hline Computational Research (a) & 0 & 0 & 0 & 0 & 0 & 0 & 0 \\
\hline NERSC Center (a) & 0 & 0 & 0 & 0 & 0 & 0 & 0 \\
\hline Information Technology (a) & 2,384 & 0 & 0 & 0 & 2,384 & 1.007 & 3,391 \\
\hline Environmental Energy Technologies & 24,583 & 1.596 & 6,549 & 12,406 & 45,135 & 628 & 45.763 \\
\hline Engineering & 3,086 & 144 & 428 & 580 & 4,238 & 4,192 & 8,429 \\
\hline EH\&S & 5,397 & 0 & 0 & 0 & 5,397 & 96 & 5.493 \\
\hline Earth Sciences & 17,466 & 6,486 & 1,838 & 3,886 & 29,676 & 180 & 29.856 \\
\hline Facilities & 1,772 & 0 & 0 & 0 & 1,772 & 10,473 & 12,244 \\
\hline Genomics & 2,546 & 0 & 7.739 & 88 & 10,373 & 64 & 10,437 \\
\hline Genomics - JGI & 40,337 & 1,843 & 371 & 1,338 & 43,890 & 1,572 & 45,461 \\
\hline Life Sciences & 10,033 & 725 & 33,766 & 7,082 & 51.006 & 923 & 51.929 \\
\hline Materials Sciences & 39,670 & 12 & 1,222 & 5,292 & 46,196 & 4,461 & 50,657 \\
\hline Nuclear Science & 15,582 & 36 & 2,563 & 7,016 & 25,198 & 2,900 & 28,098 \\
\hline Physical Biosciences & 12,676 & 386 & 7,580 & 4,135 & 24,777 & 451 & 25,228 \\
\hline Physics & 19,573 & 474 & 485 & 335 & 20,868 & 9.505 & 30,373 \\
\hline Laboratory Directorate/Other & 730 & 0 & 0 & 0 & 730 & 0 & 730 \\
\hline Other $(b)$ & $(1,018)$ & 70 & 0 & 0 & $(947)$ & 0 & $(947)$ \\
\hline Division Total & 338,161 & 14,747 & 67,284 & 45,755 & 465,947 & 50,435 & 516,382 \\
\hline \multicolumn{8}{|c|}{$\begin{array}{l}\text { Note: Minor variances may occur due to rounding. } \\
\text { (a) Computational Research, NERSC Center as well as the ESnet portion of Information Technology became part of } \\
\text { Computing Sciences in FY } 2006 \text {. } \\
\text { b) Primarily Work for Other charge to offset Safeguards and Security activities. }\end{array}$} \\
\hline
\end{tabular}


Table $1.2 \mathrm{c}$

\section{Cost By Direct Funding Source by Division, FY2006 (\$K)}

\begin{tabular}{|c|c|c|c|c|c|c|c|}
\hline \multirow[b]{2}{*}{ Division } & \multicolumn{7}{|c|}{ FY2006 } \\
\hline & $\begin{array}{c}\text { DOE } \\
\text { Operating }\end{array}$ & $\begin{array}{c}\text { DOE Integrated } \\
\text { Contractors } \\
\text { Costs }\end{array}$ & $\begin{array}{c}\text { WFO } \\
\text { Federal }\end{array}$ & $\begin{array}{l}\text { WFO Non- } \\
\text { Federal }\end{array}$ & $\begin{array}{c}\text { Operating } \\
\text { Subtotal }\end{array}$ & $\begin{array}{l}\text { Capital \& } \\
\text { Equipment }\end{array}$ & Total \\
\hline Accelerator \& Fusion Research & 18.506 & 499 & 2,054 & 3,049 & 24,108 & 1,487 & 25,595 \\
\hline Advanced Light Source & 36,269 & 80 & 0 & 928 & 37,377 & 6,803 & 44,180 \\
\hline Chemical Sciences & 11,440 & 12 & 101 & 70 & 11,623 & 931 & 12,554 \\
\hline Computing Sciences (a) & 67.768 & 4,463 & 3,129 & 489 & 75,849 & 3.765 & 79.614 \\
\hline Computational Research (a) & 0 & 0 & 0 & 0 & 0 & 0 & 0 \\
\hline NERSC Center (a) & 0 & 0 & 0 & 0 & 0 & 0 & 0 \\
\hline Information Technology (a) & 2,678 & 0 & 0 & 0 & 2,678 & 1.175 & 3,852 \\
\hline Environmental Energy Technologies & 29,091 & 1.597 & 6.991 & 14,522 & 52.201 & 851 & 53,052 \\
\hline Engineering & 1,530 & 479 & 808 & 798 & 3,615 & 1.793 & 5,408 \\
\hline EH\&S & 6,469 & 0 & 0 & 0 & 6,469 & 890 & 7,360 \\
\hline Earth Sciences & 17,932 & 6,000 & 2,777 & 3,601 & 30,310 & 727 & 31,036 \\
\hline Facilities & 3,006 & 6 & 0 & 0 & 3,011 & 28,481 & 31.492 \\
\hline Genomics & 1,759 & 0 & 7,175 & 141 & 9,075 & 658 & 9,733 \\
\hline Genomics - JGI & 35,543 & 1,71 & 1.713 & 2,410 & 40,837 & 2,268 & 43,105 \\
\hline Life Sciences & 11,153 & 5 & 29,941 & 6,607 & 47,707 & 81 & 47.788 \\
\hline Materials Sciences & 30,688 & 38 & 1,327 & 5,233 & 37,287 & 2,761 & 40,048 \\
\hline Nuclear Science & 15,536 & 74 & 1,885 & 7,423 & 24,918 & 1,583 & 26,501 \\
\hline Physical Biosciences & 12,412 & 652 & 10,606 & 4,369 & 28,039 & 1.128 & 29,167 \\
\hline Physics & 15,626 & 684 & 942 & 673 & 17,926 & 9,052 & 26,978 \\
\hline Laboratory Directorate & 754 & (2) & 0 & 0 & 752 & 0 & 752 \\
\hline Other $(\mathrm{b})$ & (887) & 0 & 0 & 0 & (887) & (173) & $(1,060)$ \\
\hline Division Total & 317,272 & 15,859 & 69.449 & 50,312 & 452,892 & 64,262 & 517.155 \\
\hline
\end{tabular}


Table $1.2 \mathrm{~d}$

\section{Cost By Direct Funding Source by Division, FY2005 (\$K)}

\begin{tabular}{|c|c|c|c|c|c|c|c|}
\hline \multirow[b]{2}{*}{ Division } & \multicolumn{7}{|c|}{ FY2005 } \\
\hline & $\begin{array}{c}\text { DOE } \\
\text { Operating }\end{array}$ & $\begin{array}{c}\text { DOE Integrated } \\
\text { Contractors } \\
\text { Costs }\end{array}$ & $\begin{array}{c}\text { WFO } \\
\text { Federal }\end{array}$ & $\begin{array}{c}\text { WFO Non- } \\
\text { Federal }\end{array}$ & $\begin{array}{l}\text { Operating } \\
\text { Subtotal }\end{array}$ & $\begin{array}{l}\text { Capital \& } \\
\text { Equipment }\end{array}$ & Total \\
\hline Accelerator \& Fusion Research & 18,917 & 592 & 2,090 & 2,051 & 23,649 & 3.513 & 27,163 \\
\hline Advanced Light Source & 34,384 & 179 & - & 917 & 35,479 & 9,545 & 45,023 \\
\hline Chemical Sciences & 11.071 & - & 164 & 74 & 11,309 & 1,042 & 12,351 \\
\hline Computing Sciences & - & - & - & 10 & 10 & - & 10 \\
\hline Computational Research & 15,346 & 911 & 2,495 & 78 & 18,828 & - & 18,828 \\
\hline NERSC Center & 30,873 & - & - & - & 30,873 & 10,426 & 41,299 \\
\hline Information Technology & 21,923 & 1,541 & 1,291 & - & 24,855 & 3,340 & 28,195 \\
\hline Environmental Energy Technologies & 29.939 & 1,343 & 6.911 & 12,801 & 50,994 & 520 & 51.514 \\
\hline Engineering & 1,256 & 446 & 842 & 1.408 & 3,952 & 552 & 4,503 \\
\hline EH\&S & 5,694 & - & - & - & 5,694 & 85 & 5,780 \\
\hline Earth Sciences & 15,341 & 7,001 & 3,055 & 2,720 & 28,117 & 837 & 28,954 \\
\hline Facilities & 1.793 & - & - & - & 1,793 & 39.481 & 41,275 \\
\hline Genomics & 739 & - & 7,048 & 415 & 8,203 & (5) & 8,198 \\
\hline Genomics - JGI & 41,283 & 0 & 611 & 1,332 & 43,227 & 3.479 & 46,706 \\
\hline Life Sciences & 9,406 & 4 & 28,614 & 4.374 & 42,398 & 715 & 43,113 \\
\hline Materials Sciences & 24,294 & 243 & 1,885 & 4,994 & 31,416 & 3,936 & 35,352 \\
\hline Nuclear Science & 16.186 & 20 & 2,067 & 8.354 & 26,627 & 2,154 & 28,781 \\
\hline Physical Biosciences & 9.982 & 432 & 12,921 & 4,096 & 27,432 & 1.248 & 28.680 \\
\hline Physics & 16,348 & 222 & 502 & 867 & 17,938 & 9,367 & 27,305 \\
\hline Laboratory Directorate & 864 & 59 & - & - & 924 & - & 924 \\
\hline Other (a) & (728) & - & - & 0 & $(728)$ & 612 & (116) \\
\hline Division Total & 304,913 & 13,092 & 70,496 & 44,490 & 432,990 & 90,847 & 523,837 \\
\hline
\end{tabular}


Table $1.2 \mathrm{e}$

\section{Cost By Direct Funding Source by Division, FY2004 (\$K)}

\begin{tabular}{|c|c|c|c|c|c|c|c|}
\hline \multirow[b]{2}{*}{ Division } & \multicolumn{7}{|c|}{ FY2004 } \\
\hline & $\begin{array}{c}\text { DOE } \\
\text { Operating }\end{array}$ & $\begin{array}{c}\text { DOE Integrated } \\
\text { Contractors } \\
\text { Costs }\end{array}$ & $\begin{array}{c}\text { WFO } \\
\text { Federal }\end{array}$ & $\begin{array}{c}\text { WFO Non- } \\
\text { Federal }\end{array}$ & $\begin{array}{l}\text { Operating } \\
\text { Subtotal }\end{array}$ & $\begin{array}{l}\text { Capital \& } \\
\text { Equip- } \\
\text { ment }\end{array}$ & Total \\
\hline Accelerator \& Fusion Research & 20,360 & 804 & 1.300 & 1.196 & 23,660 & 3,715 & 27,375 \\
\hline Advanced Light Source & 33,929 & 185 & - & 421 & 34,536 & 8,531 & 43,067 \\
\hline Chemical Sciences & 10,394 & - & 153 & 83 & 10,630 & 1,948 & 12,578 \\
\hline Computing Sciences & - & - & - & - & - & - & $=$ \\
\hline Computational Research & 16,697 & 918 & 2,066 & 85 & 19,767 & (0) & 19,767 \\
\hline NERSC Center & 28,038 & - & - & - & 28,038 & 1,432 & 29,470 \\
\hline Information Technology & 19,397 & 3,331 & 1.131 & - & 23,859 & 2,344 & 26,203 \\
\hline Environmental Energy Technologies & 34.965 & 1,353 & 6.159 & 11.342 & 53,819 & 438 & 54,257 \\
\hline Engineering & 1,226 & 405 & 266 & 1,951 & 3,847 & 710 & 4,557 \\
\hline EH\&S & 6.107 & 9 & - & - & 6,115 & 147 & 6,262 \\
\hline Earth Sciences & 13,465 & 10,626 & 2,537 & 2,664 & 29.291 & 430 & 29.721 \\
\hline Facilities & 3,523 & - & & (1) & 3,522 & 6,528 & 10,050 \\
\hline Genomics & 803 & - & 7,692 & 451 & 8,946 & 10 & 8,956 \\
\hline Genomics - JGI & 38,941 & - & 284 & 1.092 & 40,317 & 9,819 & 50,136 \\
\hline Life Sciences & 10,077 & 45 & 27,102 & 4,653 & 41,876 & 208 & 42,084 \\
\hline Materials Sciences & 25,092 & 958 & 3,814 & 5,493 & 35,356 & 16,124 & 51,481 \\
\hline Nuclear Science & 16,379 & - & 2,052 & 569 & 19,000 & 2,676 & 21,676 \\
\hline Physical Biosciences & 10,327 & 710 & 15.669 & 3,774 & 30,480 & 1,212 & 31.692 \\
\hline Physics & 14,721 & 245 & 604 & 7,085 & 22,655 & 11,150 & 33,805 \\
\hline Lab Directorate & 664 & - & - & - & 664 & - & 664 \\
\hline Other (a) & (578) & - & - & - & $(578)$ & 746 & 168 \\
\hline Division Total & 304,527 & 19,588 & 70,828 & 40,860 & 435,802 & 68,168 & 503,969 \\
\hline
\end{tabular}




\section{Indirect Budget Costs by Division, FY2008 (\$K)}

\begin{tabular}{|c|c|c|c|c|c|c|c|c|c|c|}
\hline \multirow[b]{2}{*}{ Division } & \multicolumn{3}{|c|}{ Distributed Support } & \multicolumn{6}{|c|}{ Institutional Costs } & \multirow[b]{2}{*}{ Total (a) } \\
\hline & $\begin{array}{l}\text { Org. } \\
\text { Burden }\end{array}$ & $\begin{array}{l}\text { Service } \\
\text { Centers } \\
\text { (a) (b) }\end{array}$ & $\begin{array}{l}\text { Other } \\
\text { (b) (c) }\end{array}$ & LDRD & IGPP & G\&A & $\begin{array}{c}\text { Procurement } \\
\text { Burden }\end{array}$ & $\begin{array}{c}\text { Site } \\
\text { Support }\end{array}$ & $\begin{array}{l}\text { Travel } \\
\text { Burden }\end{array}$ & \\
\hline $\begin{array}{l}\text { Accelerator \& Fusion } \\
\text { Research }\end{array}$ & 1,553 & 146 & 158 & 2,264 & $=$ & - & - & - & - & 4,121 \\
\hline $\begin{array}{l}\text { Advanced Light } \\
\text { Source }\end{array}$ & 1.911 & 193 & - & 1.319 & - & - & - & - & - & 3,423 \\
\hline $\begin{array}{l}\text { Chief Financial Officer } \\
\text { Organization }\end{array}$ & $=$ & $=$ & $=$ & $=$ & $=$ & 7,405 & 7.584 & $=$ & 1,408 & 16,397 \\
\hline Chemical Sciences & 952 & - & - & 741 & - & - & - & - & - & 1,693 \\
\hline Computing Sciences & 4,513 & - & - & 2,547 & - & - & - & - & - & 7,060 \\
\hline $\begin{array}{l}\text { Information } \\
\text { Technology }\end{array}$ & 2,795 & 6,777 & - & - & - & 13,150 & 180 & 7.667 & - & 30,570 \\
\hline $\begin{array}{l}\text { Environmental Energy } \\
\text { Technologies }\end{array}$ & 3,200 & 1,113 & - & 1.793 & - & - & - & - & - & 6,106 \\
\hline Engineering & 4,701 & 1.456 & - & (2) & + & 1,127 & - & 1,278 & - & 8,560 \\
\hline EH\&S & - & - & - & - & - & - & - & 20,199 & - & 20,199 \\
\hline Earth Sciences & 2,646 & - & - & 1.923 & + & - & - & - & - & 4,569 \\
\hline Facilities & 3,368 & 9.575 & - & - & - & - & 2,163 & 33,240 & + & 48,347 \\
\hline Genomics & 480 & - & - & - & - & - & - & - & - & 480 \\
\hline Genomics-JGI & - & - & - & 261 & - & - & - & - & - & 261 \\
\hline Laboratory Directorate & - & - & - & - & - & 10,911 & - & - & - & 10,911 \\
\hline Life Sciences & 4,390 & 499 & - & 1,717 & - & - & - & - & - & 6,606 \\
\hline Materials Sciences & 2,942 & 272 & - & 1.428 & - & $=$ & $=$ & - & - & 4,642 \\
\hline Nuclear Science & 1,336 & - & - & 1.092 & - & - & - & - & - & 2,428 \\
\hline ALD for Operations & - & 1,294 & - & - & 2,452 & 7,945 & - & 4,511 & - & 16,202 \\
\hline Physical Biosciences & 1,982 & 1.557 & - & 934 & - & - & - & - & - & 4,474 \\
\hline Physics & 1,308 & - & - & 2,236 & - & - & - & - & - & 3,544 \\
\hline Other (d) & - & - & 947 & - & - & 6,030 & - & - & - & 6,977 \\
\hline Division Total & 38,079 & 22,883 & 1,105 & 18,252 & 2,452 & 46,568 & 9,928 & 66,896 & 1,408 & 207,571 \\
\hline \multicolumn{11}{|c|}{$\begin{array}{l}\text { Note: Minor variances may occur due to rounding. } \\
\text { (a) Summation of indirect budget costs provided only to show magnitude of dollars being managed and does not equate to } \\
\text { total indirect costs since there are overlaps between indirect budgets. For example, some organization burden costs are } \\
\text { included in G\&A and Recharges. } \\
\text { (b) Service Centers includes recharge cost centers that default to B\&R YNOT (project type OHRCH) only. } \\
\text { (c) Includes: LBNL's Office of Homeland Security (formerly known as Nuclear Non-Proliferation) and Safeguards and Security } \\
\text { (S\&S). } \\
\text { (d) Includes: Work for Other charge to offset Safeguards and Security activities, and UC Management Fee. }\end{array}$} \\
\hline
\end{tabular}


Table 1.4

\section{Average FTE Breakdown by Division, FY2008 (\$K)}

\begin{tabular}{|c|c|c|c|c|c|c|c|c|c|}
\hline \multirow[b]{2}{*}{ Division } & \multicolumn{4}{|c|}{ Direct Funded FTEs } & \multicolumn{4}{|c|}{ Indirect Funded FTEs } & \multirow[b]{2}{*}{$\begin{array}{l}\text { Total } \\
\text { FTEs }\end{array}$} \\
\hline & $\begin{array}{c}\text { DOE } \\
\text { Operating } \\
\text { (a) }\end{array}$ & $\begin{array}{l}\text { WFO } \\
\text { (b) }\end{array}$ & $\begin{array}{l}\text { Capital \& } \\
\text { Equipment }\end{array}$ & $\begin{array}{c}\text { Direct } \\
\text { Funded } \\
\text { Total }\end{array}$ & $\begin{array}{l}\text { Org. } \\
\text { Burden }\end{array}$ & $\begin{array}{c}\text { Service } \\
\text { Centers } \\
\text { (c) }\end{array}$ & $\begin{array}{l}\text { Operations } \\
\text { Overhead } \\
\text { (d) }\end{array}$ & $\begin{array}{l}\text { Indirect } \\
\text { Funded } \\
\text { Total }\end{array}$ & \\
\hline $\begin{array}{l}\text { Accelerator \& Fusion } \\
\text { Research }\end{array}$ & 73.5 & 7.1 & 9.3 & 89.9 & 10.8 & 0.4 & 9.8 & 21.0 & 110.9 \\
\hline $\begin{array}{l}\text { Advanced Light } \\
\text { Source }\end{array}$ & 177.6 & 0.1 & 10.4 & 188.2 & 13.1 & 0.3 & 6.9 & 20.3 & 208.5 \\
\hline $\begin{array}{l}\text { Chief Financial Officer } \\
\text { Organization }\end{array}$ & 0.1 & $=$ & $=$ & 0.1 & $=$ & $=$ & 131.1 & 131.1 & 131.2 \\
\hline Chemical Sciences & 66.0 & 1.9 & 0.1 & 68.1 & 6.6 & - & 4.1 & 10.7 & 78.8 \\
\hline Computing Sciences & 144.3 & 6.2 & - & 150.5 & 33.8 & - & 10.1 & 43.9 & 194.4 \\
\hline $\begin{array}{l}\text { Environmental Energy } \\
\text { Technologies }\end{array}$ & 98.7 & 55.6 & - & 154.4 & 24.4 & 9.3 & 5.9 & 39.6 & 194.0 \\
\hline Engineering & 0.7 & 2.7 & 8.1 & 11.5 & 30.1 & 8.0 & 8.0 & 46.1 & 57.6 \\
\hline EH\&S & 5.1 & - & - & 5.1 & - & - & 103.8 & 103.8 & 108.9 \\
\hline Earth Sciences & 83.6 & 21.9 & 1.3 & 106.8 & 14.5 & - & 5.9 & 20.4 & 127.1 \\
\hline Facilities & 4.6 & - & 8.8 & 13.3 & 21.7 & 4.3 & 146.5 & 172.5 & 185.8 \\
\hline Genomics & 9.0 & 28.2 & & 37.2 & 4.6 & & & 4.6 & 41.7 \\
\hline Genomics - JGl & 155.4 & 5.8 & & 161.2 & & & 1.9 & 1.9 & 163.1 \\
\hline $\begin{array}{l}\text { Information } \\
\text { Technology }\end{array}$ & 7.6 & - & - & 7.6 & 15.1 & 26.5 & 91.6 & 133.2 & 140.7 \\
\hline Laboratory Directorate & 0.2 & - & - & 0.2 & - & - & 54.3 & 54.3 & 54.5 \\
\hline Life Sciences & 54.3 & 176.0 & - & 230.2 & 37.4 & 3.0 & 8.4 & 48.7 & 279.0 \\
\hline Materials Sciences & 191.4 & 33.0 & 2.3 & 226.7 & 20.8 & 1.7 & 6.1 & 28.7 & 255.4 \\
\hline Nuclear Science & 73.9 & 19.6 & 5.5 & 99.0 & 9.8 & + & 5.1 & 14.8 & 113.8 \\
\hline ALD for Operations & 2.1 & - & - & 2.1 & - & 11.0 & 70.3 & 81.2 & 83.3 \\
\hline Physical Biosciences & 83.1 & 31.4 & 0.2 & 114.7 & 14.9 & 0.1 & 6.2 & 21.2 & 135.9 \\
\hline Physics & 72.9 & 5.4 & 20.1 & 98.4 & 9.1 & - & 9.0 & 18.1 & 116.6 \\
\hline Division Total & $1,304.0$ & 395.0 & 66.1 & $1,765.2$ & 266.7 & 64.5 & 685.0 & $1,016.2$ & $2,781.4$ \\
\hline \multicolumn{10}{|c|}{$\begin{array}{l}\text { Notes: } \\
\text { - Minor variances may occur due to rounding. } \\
\text { Factor (PLF). } \\
\text { - FTE calculation does not include Contract Labor or Campus Labor. } \\
\text { - Total FTE excludes } 43.8 \text { FTEs from "Funds Held for Others Costs" projects (see table } 1.5 \text { for examples). } \\
\text { (a) DOE Operating includes DOE Integrated Contractors, Conferences and Fellowships. } \\
\text { (b) WFO includes CRADA. } \\
\text { (c) Service Centers includes recharge cost centers that default to B\&R YNOI (project type OHRCH) only. } \\
\text { (d) Operations Overhead includes: G\&A, Site Support, LDRD, Institutional General Plant Projects (IGPP), Procurement, Travel, } \\
\text { Payroll Burden, S\&S, and LBNL's Office of Homeland Security. }\end{array}$} \\
\hline
\end{tabular}


Table 1.5

\section{Funds Held for Others' Costs, FY2004 - FY2008 (\$K)}

\begin{tabular}{|c|c|c|c|c|c|}
\hline Funding Source & FY2004 & FY2005 & FY2006 & FY2007 & FY2008 \\
\hline Royalty & 729 & 1,274 & 1,976 & 2,121 & 1,677 \\
\hline Contractor Supporting Research (CSR) & 0 & 0 & 151 & 2,113 & 2,054 \\
\hline Gifts & 0 & 0 & 267 & 429 & 889 \\
\hline Inter Location Appointments (ILA) & 0 & 0 & 0 & 940 & 2,965 \\
\hline UC Construction Projects & 0 & 0 & 0 & 900 & 1,173 \\
\hline University of California Directed Research and Development (UCDRD) & 1,657 & 2,604 & 165 & 0 & 0 \\
\hline UC Bid Contract Competition & 373 & 1,847 & 0 & 0 & 0 \\
\hline Other & 30 & 16 & 85 & 44 & 304 \\
\hline Total & 2,789 & 5,741 & 2,644 & 6,548 & 9,061 \\
\hline
\end{tabular}


2. Direct Funding - DOE and Reimbursable Work 


\section{Direct Funding - DOE and Reimbursable Work}

\section{Total Laboratory Funding - Increase $\$ 47.5 \mathrm{M}$}

Total funding increased $\$ 47.5 \mathrm{M}$ to a total of $\$ 617.6 \mathrm{M}$. This increase was primarily for DOE operating and maintenance projects in the amount of $\$ 58.8 \mathrm{M}$. Funding for DOE construction projects increased $\$ 7.4 \mathrm{M}$ and overall funding from other non-DOE sponsors decreased by $\$ 18.7 \mathrm{M}$.

\section{DOE Operating and Maintenance Funding - Increase $\$ 58.8 \mathrm{M}$}

DOE operating and maintenance (O\&M) new funding (budget authority) provided to the Laboratory totaled $\$ 487 \mathrm{M}$ in FY2008, an increase of $\$ 58.8 \mathrm{M}$ from FY2007. O\&M funding provides for the execution of direct operations, the purchase of equipment and the construction of general plant projects. While we experienced some minor fluctuation in funding levels provided by the various Assistant Secretarial Offices, the FY2008 increase was provided almost entirely by the Office of Science. O\&M funding provided by the Office of Science represents an increase of $16 \%$ over FY2007 funding levels.

$\$ 26.4 \mathrm{M}$ of the increase was provided by the Advanced Scientific Computing Research program (ASCR) in support of NERSC and EsNet. This additional funding was made available for the NERSC 5 system procurement and related costs, as well as the Science Data Network deployment and equipment upgrades.

$\$ 22.2 \mathrm{M}$ can be attributed to the Biological and Environmental Research (BER) program and was due in large part to the $\$ 17 \mathrm{M}$ increase provided for the support of various DOE BioEnergy Research Centers, including $\$ 15 \mathrm{M}$ for the Joint BioEnergy Institute (JBEI). Also contributing to the increase was $\$ 3.8 \mathrm{M}$ for the purchase of sequencers at the Joint Genome Institute (JGI), as well as $\$ 2.4 \mathrm{M}$ for new efforts in Radiopharmaceuticals \& Imaging and \$1.5M for new efforts in Low Dose. These increases were partially offset by a decrease related to the lease renewal at the JGI which was funded in FY2007.

Additionally, a $\$ 4.3 \mathrm{M}$ increase was provided for the Bevatron Demolition. This facility disposition project appropriately disposes of Building 51 and the accelerator in order to free the site to make use of excess, under-utilized space. It is a multi-year project that is currently planned for completion in FY2012.

\section{DOE Construction Funding - Increase $\$ 7.4 \mathrm{M}$}

Line-item construction funding provided by the Office of science increased in FY2008 to $\$ 14.2 \mathrm{M}$. The funding for the Seismic Safety Building Replacement effort increased by $\$ 4.2 \mathrm{M}$, and the funding for the Advanced Light Source User Support Building increased by $\$ 3.5 \mathrm{M}$. Both are multiyear projects that moved into the construction phase in FY2008. This increase is partially offset by the completion of the Molecular Foundry building, which received it's final funding increment in $\mathrm{FY} 2007$ ( $\$ 0.3 \mathrm{M})$.

\section{Other Direct Operating Funding - Decrease $\$ 18.7 M$}

In FY2008, overall funding from DOE Integrated Contractors and non-DOE Work-for-Others sponsors decreased by $\$ 18.7 \mathrm{M}$ in total. A $\$ 4.5 \mathrm{M}$ decrease in DOE Integrated Contractor funding and $a \$ 21.5 \mathrm{M}$ decrease in funding from Other Federal Agencies was offset by: $a \$ 5.4 \mathrm{M}$ increase in funding from non-Federal sponsors, a $\$ 2.0 \mathrm{M}$ increase in funding of Cooperative Research, and Development Agreements (CRADAs).

DOE Integrated Contractor funding decreased $\$ 4.5 \mathrm{M}$ or $30 \%$ in FY2008. This decrease is attributed to a shift in work scope for the Yucca Mountain Project from scientific investigation in FY2007 to license application in FY2008.

Funding from Other Federal Agencies decreased $\$ 21.5 \mathrm{M}$ or $26 \%$ in FY2008. This was primarily due to a $\$ 14.2 \mathrm{M}$ decrease in funding from the National Institutes of Health $(\mathrm{N} \mid \mathrm{H})$ and an $\$ 8.2 \mathrm{M}$ decrease in funding from the Department of Defense (DOD). A portion of the NIH decrease was due to timing whereby, $\$ 8.9 \mathrm{M}$ in funding agreements were received immediately after the DOE's FY2006 processing deadlines. As a result, funding appeared higher in FY2007 and then dropped back down in FY2008. The remaining $\$ 5.3 \mathrm{M}$ decrease in $\mathrm{NIH}$ funding is a result of various decreases throughout the Genomics and Physical Biosciences Divisions, the largest impact was due to a Physical Biosciences Division NIH Eye Institute grant being renewed through the University of California, Berkeley. The apparent decrease in funding from the DOD is aftributed to LBNL's Life Sciences Division receipt of a five year breast cancer research grant in FY2007. The entire five-year funding outlay was received in FY2007, inflating FY2007 funding levels when compared to FY2008. 


\section{Direct Funding - DOE and Reimbursable Work Continued}

Non-Federal funding increased $\$ 5.4 \mathrm{M}$ or $14 \%$ in FY2008.

The increase in funding was spread across state and local

governments, non-profit organizations, and industry.

Data sources for tables in this section are as follows:

\begin{tabular}{|l|l|}
\hline Data Type & Source \\
\hline FY2008 Beginning Uncosted Obligations & Carryover Funding as provided in the LBNL final FY2008 Contract Modification (GSO) \\
\hline FY2008 Funds & Budget Authority as provided in the LBNL contract modification for the fiscal year \\
\hline FY2008 Costs & LBNL published Fiscal Year End Costs \\
\hline FY2008 Ending Uncosted Obligations & $\begin{array}{l}\text { DOE - Beginning Uncosted + Funds - Costs } \\
\text { WFO- The sum of FY2008 Beginning Uncosted, and FY2008 Funds, minus FY2008 Costs } \\
\text { for the "Other Direct Operating" categories does not equal FY2008 Ending Uncosted } \\
\text { Obligations due to various adjustments not reflected in the FY2008 Costs column. Exam- } \\
\text { ples of these adjustments include Bridge Funding, suspense items, and Federal Admin- } \\
\text { istrative Charge. The total of these adjustments for FY2008 is (\$0.6M). }\end{array}$ \\
\hline
\end{tabular}


Table 2.1

\section{LBNL Fund Trends (BA) by Funding Source (\$K)}

\begin{tabular}{|c|c|c|c|c|c|}
\hline LBNL Fund Trends (BA) by funding source (\$K) & FY2004 & FY2005 & FY2006 & FY2007 & FY2008 \\
\hline \multicolumn{6}{|l|}{ DOE DIRECT OPERATING } \\
\hline Administrator for National Nuclear Security Administration & 7,344 & 4,712 & 6,045 & 5,387 & 5.179 \\
\hline Assistant Secretary for Energy Efficiency and Renewable Energy & 25,885 & 26,701 & 20,516 & 26,520 & 27,102 \\
\hline Assistant Secretary for Environmental Management & 2,784 & 4,037 & 3,861 & 1.709 & - \\
\hline Assistant Secretary for Fossil Energy & 5,491 & 5,859 & 7,017 & 6,328 & 8,124 \\
\hline Assistant Secretary for Nuclear Energy & - & - & - & - & 788 \\
\hline Assistant Secretary for Policy and International Affairs & - & -1 & 10 & (0) & 65 \\
\hline Office of Civilian Radioactive Waste Management & 1.643 & 3,151 & 2,331 & 1,387 & (0) \\
\hline Office of Electricity Delivery and Energy Reliability & 5,632 & 4,500 & 4,486 & 7.213 & 4.970 \\
\hline Office of Energy and Threat & - & -1 & - & - & 65 \\
\hline Office of Health Safety and Security & 465 & 724 & 611 & 564 & 413 \\
\hline Office of Intelligence & 181 & - & - & - & - \\
\hline Office of Science & 249,368 & 267.062 & 299,606 & 329,097 & 371,361 \\
\hline Office of the Chief Financial Officer & - & - & (1) & - & - \\
\hline Office of the Chief Information Officer & 538 & - & (0) & - & - \\
\hline Technical Analysis & - & 220 & - & (0) & $=$ \\
\hline Total DOE Direct Operating & 299,331 & 316,966 & 344,482 & 378,206 & 418,067 \\
\hline \multicolumn{6}{|l|}{ OTHER DIRECT OPERATING } \\
\hline Work for Other Federal Agencies & 76,360 & 71.879 & 60,209 & 83,164 & 61,640 \\
\hline Work for Non-Federal Sponsors (a) & 42,947 & 48,036 & 57,078 & 38,529 & 43,882 \\
\hline Cooperative Research and Development Agreements & 387 & 554 & 633 & $(1.419)$ & 539 \\
\hline Work for Other DOE Integrated Contractors (b) & 16,771 & 13,092 & 15,859 & 14,747 & 10,296 \\
\hline Total Other Direct Operating & 136,465 & 133,561 & 133,779 & 135,020 & 116,357 \\
\hline TOTAL OPERATING & 435,796 & 450,526 & 478,260 & 513,226 & 534,424 \\
\hline
\end{tabular}


Table 2.1

\section{LBNL Fund Trends (BA) by Funding Source (\$K) Continued}

\begin{tabular}{|c|c|c|c|c|c|}
\hline LBNL Fund Trends (BA) by funding source (\$K) & FY2004 & FY2005 & FY2006 & FY2007 & FY2008 \\
\hline \multicolumn{6}{|l|}{ DOE PLANT AND CAPITAL EQUIPMENT } \\
\hline \multicolumn{6}{|l|}{ Basic Equipment/Major Items of Equipment } \\
\hline Administrator for National Nuclear Security Administration & - & - & 450 & 340 & 2,205 \\
\hline Assistant Secretary for Energy Efficiency and Renewable Energy & 543 & 400 & 345 & 496 & 1.097 \\
\hline Assistant Secretary for Environmental Management & - & - & - & (0) & - \\
\hline Assistant Secretary for Fossil Energy & 50 & - & (8) & $(0)$ & - \\
\hline Office of Electricity Delivery and Energy Reliability & - & - & - & - & - \\
\hline Office of Intelligence & - & (2) & - & - & - \\
\hline Office of Science & 51.272 & 47,508 & 33,211 & 41.243 & 58,836 \\
\hline Total & 51,864 & 47,906 & 33,998 & 42,079 & 62,138 \\
\hline \multicolumn{6}{|l|}{ General Plant Projects } \\
\hline Office of Science & 3,500 & 4,765 & 4,864 & 4,031 & 4,775 \\
\hline \multicolumn{6}{|l|}{ Accelerator Improvement Projects } \\
\hline Office of Science & 1,800 & 4,000 & 1.200 & 3,866 & 2,050 \\
\hline \multicolumn{6}{|l|}{ Line-Ifem Construction } \\
\hline Administrator for National Nuclear Security Administration & - & - & - & (1) & - \\
\hline Assistant Secretary for Energy Efficiency and Renewable Energy & - & $(10)$ & - & - & - \\
\hline Office of Science & 36,882 & 37,673 & 13,290 & 6,868 & 14,226 \\
\hline Total & 36,882 & 37,663 & 13,290 & 6,867 & 14,226 \\
\hline TOTAL DOE PLANT AND CAPITAL EQUIPMENT & 94,046 & 94,334 & 53,352 & 56,843 & 83,189 \\
\hline TOTAL LABORATORY & 529,843 & 544,860 & 531,612 & 570,069 & 617,613 \\
\hline \multicolumn{6}{|c|}{$\begin{array}{l}\text { Note: Minor variances may accur due to rounding. } \\
\text { Data Source: Budget Authority as provided in the LBNL final contract mo dification for the fiscal year. } \\
\text { (a) Includes funding for Non-Federal Sponsors who are precluded by law from paying an advance under the WNO2 program. } \\
\text { (b) Total funding for Work for Other Integrated Contractors is assumed to be equal to cost incurred. }\end{array}$} \\
\hline
\end{tabular}


Table 2.2

\section{LBNL Cost Trends by Funding Source (\$K)}

\begin{tabular}{|c|c|c|c|c|c|}
\hline LBNL Spending Trends by funding source (\$K) & FY2004 & FY2005 & FY2006 & FY2007 & FY2008 \\
\hline \multicolumn{6}{|l|}{ DOE DIRECT OPERATING } \\
\hline Administrator for National Nuclear Security Administration & 8,508 & 5.689 & 6,078 & 6,194 & 5,703 \\
\hline Assistant Secretary for Energy Efficiency and Renewable Energy & 28,579 & 25,844 & 22,337 & 18,050 & 24,059 \\
\hline Assistant Secretary for Environmental Management & 3,285 & 3,130 & 3,603 & 2.293 & 242 \\
\hline Assistant Secretary for Fossil Energy & 5,359 & 4,807 & 5,012 & 5.796 & 7,060 \\
\hline Assistant Secretary for Nuclear Energy & & & & & 206 \\
\hline Assistant Secretary for Policy and International Affairs & 83 & - & 4 & 6 & - \\
\hline Office of Civilian Radioactive Waste Management & 225 & 1.785 & 3,000 & 1,154 & 2,078 \\
\hline Office of Electricity Delivery and Energy Reliability & 4,087 & 3,650 & 5,761 & 4,339 & 4,242 \\
\hline Office of Energy and Threat & & & & & - \\
\hline Office of Health Safety and Security & 473 & 684 & 576 & 563 & 542 \\
\hline Office of Intelligence & 128 & 86 & - & - & - \\
\hline Office of Science & 253,439 & 258,899 & 270,841 & 299.767 & 361,416 \\
\hline Office of the Chief Financial Officer & - & - & - & - & - \\
\hline Office of the Chief Information Officer & 359 & 179 & - & - & - \\
\hline Technical Analysis & - & 161 & 59 & - & - \\
\hline Total DOE Direct Operating & 304,527 & 304,913 & 317,272 & 338,161 & 405,548 \\
\hline \multicolumn{6}{|l|}{ OTHER DIRECT OPERATING } \\
\hline Work for Other Federal Agencies & 70,828 & 70,496 & 69,449 & 67.284 & 64,195 \\
\hline Work for Non-Federal Sponsors (a) & 40,506 & 44,047 & 49.670 & 45,627 & 43,412 \\
\hline Cooperative Research and Development Agreements & 354 & 443 & 642 & 128 & 452 \\
\hline Work for Other DOE Integrated Contractors & 19,588 & 13,092 & 15,859 & 14.747 & 10,296 \\
\hline Total Other Direct Operating (b) & 131,275 & 128,077 & 135,620 & 127,786 & 118,355 \\
\hline TOTAL OPERATING & 435,802 & 432,991 & 452,892 & 465,947 & 523,904 \\
\hline
\end{tabular}

continued... 
Table 2.2

\section{LBNL Cost Trends by Funding Source (\$K) Continued}

\begin{tabular}{|c|c|c|c|c|c|}
\hline LBNL Spending Trends by funding source (\$K) & FY2004 & FY2005 & FY2006 & FY2007 & FY2008 \\
\hline \multicolumn{6}{|l|}{ DOE Plant and Capital Equipment } \\
\hline \multicolumn{6}{|l|}{ Basic Equipment/Major Items of Equipment } \\
\hline Administrator for National Nuclear Security Administration & 10 & - & 168 & 267 & 1,343 \\
\hline Assistant Secretary for Energy Efficiency and Renewable Energy & 282 & 248 & 312 & 319 & 763 \\
\hline Assistant Secretary for Environmental Management & - & - & - & - & - \\
\hline Assistant Secretary for Fossil Energy & - & 41 & 9 & - & - \\
\hline Office of Electricity Delivery and Energy Reliability & 12 & - & - & - & - \\
\hline Office of Intelligence & - & - & - & - & - \\
\hline Office of Science & 46.291 & 49,491 & 32,243 & 37,242 & 47,907 \\
\hline Total & 46,596 & 49,780 & 32,733 & 37,828 & 50,013 \\
\hline \multicolumn{6}{|l|}{ General Plant Projects } \\
\hline Office of Science & 4,127 & 1.533 & 4,135 & 6,082 & 4,340 \\
\hline \multicolumn{6}{|l|}{ Accelerator Improvement Projects } \\
\hline Office of Science & 2,610 & 1,715 & 2,453 & 2,038 & 2,680 \\
\hline \multicolumn{6}{|l|}{ Line-Ifem Construction } \\
\hline Administrator for National Nuclear Security Administration & 0 & - & - & - & - \\
\hline Assistant Secretary for Energy Efficiency and Renewable Energy & - & - & - & - & - \\
\hline Office of Science & 14,834 & 37,819 & 24,941 & 4.487 & 8,561 \\
\hline Total & 14,834 & 37,819 & 24,941 & 4,487 & 8,561 \\
\hline TOTAL DOE PLANT AND CAPITAL EQUIPMENT & 68,168 & 90,847 & 64,262 & 50,435 & 65,595 \\
\hline TOTAL LABORATORY & 503,969 & 523,837 & 517,155 & 516,382 & 589,498 \\
\hline \multicolumn{6}{|c|}{$\begin{array}{l}\text { Note: Minor variances may accur due to rounding. } \\
\text { Data Source: LBNL published Fiscal Year End Costs. } \\
\text { (a) Includes costs for Non-Federal Sponsors who are precluded by law from paying an advance under the WNO2 program. } \\
\text { (b) FY2008 Costs do not include various adjustments. Examples of these adjustments include bridge funding, suspense items } \\
\text { and Federal Administrative Charge. The total of these adjustments for FY2008 is ( } \$ 558 \mathrm{~K} \text { ). }\end{array}$} \\
\hline
\end{tabular}


Table 2.3

\section{Laboratory Funding and Costs by Source (\$K)}

\begin{tabular}{|c|c|c|c|c|}
\hline LBNL FY2008 funding and cost by source ( $\$ K)$ & $\begin{array}{l}\text { FY2008 } \\
\text { Beginning } \\
\text { Uncosted } \\
\text { Obligations }\end{array}$ & $\begin{array}{l}\text { FY2008 } \\
\text { Funds }\end{array}$ & $\begin{array}{l}\text { FY2008 } \\
\text { Costs }\end{array}$ & $\begin{array}{l}\text { FY2008 } \\
\text { Ending } \\
\text { Uncosted } \\
\text { Obligations }\end{array}$ \\
\hline \multicolumn{5}{|l|}{ DOE DIRECT OPERATING } \\
\hline Administrator for National Nuclear Security Administration & 3,640 & 5,179 & 5,703 & 3.115 \\
\hline Assistant Secretary for Energy Efficiency and Renewable Energy & 14,562 & 27,102 & 24,059 & 17.604 \\
\hline Assistant Secretary for Environmental Management & 1,214 & - & 242 & 972 \\
\hline Assistant Secretary for Fossil Energy & 8.138 & 8,124 & 7.060 & 9,202 \\
\hline Assistant Secretary for Nuclear Energy & - & 788 & 206 & 583 \\
\hline Assistant Secretary for Policy and International Affairs & - & 65 & - & 65 \\
\hline Office of Civilian Radioactive Waste Management & 2,353 & (0) & 2,078 & 274 \\
\hline Office of Electricity Delivery and Energy Reliability & 5,169 & 4,970 & 4,242 & 5,897 \\
\hline Office of Energy and Threat & - & 65 & - & 65 \\
\hline Office of Health Safety and Security & 283 & 413 & 542 & 154 \\
\hline Office of Science & 109,303 & 371,361 & 361,416 & 119,249 \\
\hline Total DOE Direct Operating & 144,661 & 418,067 & 405,548 & 157,180 \\
\hline \multicolumn{5}{|l|}{ OTHER DIRECT OPERATING } \\
\hline Work for Other Federal Agencies & 80,418 & 61,640 & 64,195 & 78,315 \\
\hline Work for Non-Federal Sponsors (a) & 24,383 & 43,882 & 43,412 & 24,947 \\
\hline Cooperative Research and Development Agreements & 235 & 539 & 452 & 335 \\
\hline Work for Other DOE Integrated Contractors (b) & - & 10,296 & 10,296 & - \\
\hline Total Other Direct Operating (c) & 105,037 & 116,357 & 118,355 & 103,597 \\
\hline TOTAL OPERATING & 249,698 & 534,424 & 523,904 & 260,776 \\
\hline
\end{tabular}




\section{Laboratory Funding and Costs by Source (\$K) Continued}

\begin{tabular}{|c|c|c|c|c|}
\hline LBNL FY2008 funding and cost by source (\$K) & $\begin{array}{l}\text { FY2008 } \\
\text { Beginning } \\
\text { Uncosted } \\
\text { Obligations }\end{array}$ & $\begin{array}{l}\text { FY2008 } \\
\text { Funds }\end{array}$ & $\begin{array}{c}\text { FY2008 } \\
\text { Costs }\end{array}$ & $\begin{array}{l}\text { FY2008 } \\
\text { Ending } \\
\text { Uncosted } \\
\text { Obligations }\end{array}$ \\
\hline \multicolumn{5}{|l|}{ DOE PLANT AND CAPITAL EQUIPMENT } \\
\hline \multicolumn{5}{|l|}{ Basic Equipment/Major Items of Equipment } \\
\hline Administrator for National Nuclear Security Administration & 355 & 2,205 & 1.343 & 1,218 \\
\hline Assistant Secretary for Energy Efficiency and Renewable Energy & 846 & 1,097 & 763 & 1,180 \\
\hline Office of Science & 35,463 & 58,836 & 47.907 & 46,392 \\
\hline Total & 36,664 & 62,138 & 50,013 & 48,789 \\
\hline \multicolumn{5}{|l|}{ General Plant Projects } \\
\hline Office of Science & 3,328 & 4,775 & 4,340 & 3,763 \\
\hline \multicolumn{5}{|l|}{ Accelerator Improvement Projects } \\
\hline Office of Science & 3,607 & 2,050 & 2,680 & 2,977 \\
\hline \multicolumn{5}{|l|}{ Line-Ifem Construction } \\
\hline Office of Science & 17,592 & 14,226 & 8,561 & 23,256 \\
\hline TOTAL DOE PLANT AND CAPITAL EQUIPMENT & 61,191 & 83,189 & 65,595 & 78,785 \\
\hline TOTAL LABORATORY & 310,889 & 617,613 & 589,498 & 339,562 \\
\hline \multicolumn{5}{|c|}{$\begin{array}{l}\text { Note: Minor variances may occur due to rounding. } \\
\text { (a) Includes funding for Non-Federal Sponsors who are precluded by law from paying an advance under the WN02 program. } \\
\text { (b) Total funding for Work for Other Integrated Contractors is assumed to be equal to cost incurred. } \\
\text { (c) The sum of FY2008 Beginning Uncosted Obligations and FY2008 Funds, minus, FY2008 Costs does not equal FY2008 Ending } \\
\text { Uncosted Obligations due to various adjustments not reflected in the FY2008 Costs column. Examples of these adjustments include } \\
\text { bridge funding, suspense items, and Federal Administrative Charge. The total of these adjustments for FY2008 is (\$558K). }\end{array}$} \\
\hline
\end{tabular}




\section{DOE Programs (\$K)}

\begin{tabular}{|c|c|c|c|c|c|}
\hline \multicolumn{2}{|c|}{ Administrator for National Nuclear Security Administration } & $\begin{array}{c}\text { FY2008 } \\
\text { Beginning } \\
\text { Uncosted } \\
\text { Obligations }\end{array}$ & $\begin{array}{l}\text { FY2008 } \\
\text { Funds }\end{array}$ & $\begin{array}{l}\text { FY2008 } \\
\text { Costs }\end{array}$ & $\begin{array}{l}\text { FY2008 Ending } \\
\text { Uncosted } \\
\text { Obligations }\end{array}$ \\
\hline \multicolumn{6}{|l|}{ OPERATING: } \\
\hline DP12 & Science Campaign & 220 & 160 & 380 & 0 \\
\hline DP15 & Advanced Simulation and Computing Campaign & - & 10 & 10 & 0 \\
\hline FS21 & Cyber Security & - & 180 & 180 & 0 \\
\hline NN2O & $\begin{array}{l}\text { Nonproliferation and Verification Research and } \\
\text { Development }\end{array}$ & 756 & 4,640 & 3,829 & 1,566 \\
\hline NN40 & Nonproliferation and International Security & 599 & 215 & 388 & 425 \\
\hline NN41 & Global Initiative for Proliferation Prevention & 2,040 & - & 917 & 1.123 \\
\hline PSO3 & NNSA Information Technology & 26 & (26) & - & - \\
\hline \multicolumn{2}{|r|}{ Total Operating } & 3,640 & 5,179 & 5,703 & 3,115 \\
\hline \multicolumn{6}{|c|}{ CAPITALEQUIPMENT: } \\
\hline NN2O & $\begin{array}{l}\text { Nonproliferation and Verification Research and } \\
\text { Development }\end{array}$ & 355 & 2,205 & 1,343 & 1.218 \\
\hline \multicolumn{2}{|r|}{ Total Capital Equipment } & 355 & 2,205 & 1,343 & 1,218 \\
\hline \multicolumn{2}{|c|}{ Total Administrator for National Nuclear Security Administration } & 3,995 & 7,384 & 7.046 & 4,333 \\
\hline
\end{tabular}


Table 2.4

DOE Programs ( $\mathbf{K}$ ) Continued

\begin{tabular}{|c|c|c|c|c|c|}
\hline \multicolumn{2}{|c|}{ Office of Science } & $\begin{array}{c}\text { FY2008 } \\
\text { Beginning } \\
\text { Uncosted } \\
\text { Obligations }\end{array}$ & $\begin{array}{l}\text { FY2008 } \\
\text { Funds }\end{array}$ & $\begin{array}{c}\text { FY2008 } \\
\text { Costs }\end{array}$ & $\begin{array}{l}\text { FY2008 } \\
\text { Ending } \\
\text { Uncosted } \\
\text { Obligations }\end{array}$ \\
\hline \multicolumn{6}{|c|}{ OPERATING: } \\
\hline AT50 & Fusion Energy Sciences - Science & 583 & 4,796 & 4,924 & 455 \\
\hline FS10 & Safeguards and Security - Science & 649 & 4,004 & 4,214 & 440 \\
\hline KA11 & Proton Accelerator-Based Physics: & 832 & 10,694 & 10,477 & 1,050 \\
\hline KA12 & Electron Accelerator-Based Physics & 527 & 770 & 1,159 & 137 \\
\hline KA13 & Non-Accelerator-Based Physics & 1,536 & 10,861 & 8,449 & 3,948 \\
\hline KA 14 & Theoretical Physics & 2,823 & 5,012 & 4,926 & 2,909 \\
\hline KA15 & Advanced Technology R\&D & 2,623 & 10,338 & 10,392 & 2,568 \\
\hline $\mathrm{KBO} 2$ & Heavy-Ion Physics & 2,925 & 6,308 & 5,666 & 3,567 \\
\hline KBO3 & Nuclear Theory & 418 & 1.994 & 1,942 & 470 \\
\hline KBO4 & Low Energy Physics & 2,662 & 9,684 & 9,600 & 2,745 \\
\hline $\mathrm{KCO} 2$ & Materials Sciences and Engineering & 13,808 & 89,520 & 86,827 & 16.501 \\
\hline $\mathrm{KCO3}$ & Chemical Sciences, Geosciences, and Energy Biosc ences & 9,332 & 22,407 & 22,966 & 8,773 \\
\hline KCO6 & Excess Facilities Disposition & 12,809 & 8.145 & 4,145 & 16,809 \\
\hline KG08 & Safety-Related Corrective Actions & 4 & - & - & 4 \\
\hline KJOI & Mathematical, Information, And Computational Sciences & 17,432 & 84,910 & 85,517 & 16,826 \\
\hline KLO1 & Undergraduate Research Internships & 137 & 359 & 318 & 178 \\
\hline KLO2 & Graduate/Faculty Fellowships & 344 & 276 & 305 & 314 \\
\hline KPII & Life Sciences & 36,184 & $(1,425)$ & 19,805 & 14,954 \\
\hline KP12 & Climate Change Research & 1.066 & 3,186 & 2,647 & 1,605 \\
\hline KP13 & Environmental Remediation & 2,226 & (1) & 1,338 & 887 \\
\hline KP14 & Medical Applications and Measurement Science & 383 & - & 333 & 50 \\
\hline KP15 & Biological Research & - & 99,523 & 75,465 & 24,057 \\
\hline \multicolumn{2}{|r|}{ Total Operating } & 109,303 & 371,361 & 361,416 & 119,249 \\
\hline \multicolumn{6}{|c|}{ CAPITALEQUIPMENT: } \\
\hline AT50 & Fusion Energy Sciences - Science & 58 & - & 56 & 2 \\
\hline FS10 & Safeguards and Security-Science & - & 160 & 152 & 8 \\
\hline KA11 & Proton Accelerator-Based Physics (a) & 392 & 3.699 & 3,635 & 456 \\
\hline KA13 & Non-Accelerator-Based Physics & 476 & 3,480 & 2,130 & 1,826 \\
\hline KA 15 & Advanced Technology R\&D & 2,628 & 4,389 & 3,959 & 3,058 \\
\hline $\mathrm{KBO} 2$ & Heavy-Ion Physics & 783 & 2,200 & 1.778 & 1.205 \\
\hline
\end{tabular}




\section{DOE Programs (\$K) Continued}

\begin{tabular}{|c|c|c|c|c|c|}
\hline \multicolumn{2}{|c|}{ Office of Science (Continued) } & \multirow{2}{*}{$\begin{array}{c}\text { FY2008 } \\
\text { Beginning } \\
\text { Uncosted } \\
\text { Obligations } \\
5,375\end{array}$} & \multirow{2}{*}{$\begin{array}{l}\begin{array}{l}\text { FY2008 } \\
\text { Funds }\end{array} \\
4,570\end{array}$} & \multirow{2}{*}{$\begin{array}{r}\begin{array}{l}\text { FY2008 } \\
\text { Costs }\end{array} \\
1,797\end{array}$} & \multirow{2}{*}{$\begin{array}{c}\begin{array}{c}\text { FY2008 } \\
\text { Ending } \\
\text { Uncosted } \\
\text { Obligations }\end{array} \\
8,147\end{array}$} \\
\hline KBO4 & Low Energy Physics & & & & \\
\hline $\mathrm{KCO} 2$ & Materials Sciences and Engineering & 18,598 & 10,350 & 17,127 & 11,821 \\
\hline $\mathrm{KCO3}$ & Chemical Sciences, Geosciences, and Energy Biosc ences & 2,786 & 1,740 & 2,578 & 1,947 \\
\hline KJOI & Mathematical, Information, And Computational Sciences & 1.851 & 17,200 & 8,440 & 10,611 \\
\hline KP11 & Life Sciences & 2,130 & 1,177 & 2,508 & 798 \\
\hline KP12 & Climate Change Research & 4 & - & - & 4 \\
\hline KP13 & Environmental Remediation & 378 & - & 225 & 153 \\
\hline KP14 & Medical Applications and Measurement Science & 4 & (1) & - & 3 \\
\hline KP15 & Biological Research & - & 9,872 & 3,521 & 6,350 \\
\hline \multicolumn{2}{|r|}{ Total Capital Equipment } & 35,463 & 58,836 & 47,907 & 46,392 \\
\hline \multicolumn{6}{|c|}{$\begin{array}{l}\text { Note: Minor variances may occur due to rounding. Sub-program titles may have changed since publication of the } \\
\text { FY2007 Annual Report. } \\
\text { (a) Includes Institutional General Purpose Equipment activity. }\end{array}$} \\
\hline \multicolumn{6}{|c|}{ ACCELERATOR IMPROVEMENT PROJECTS: } \\
\hline $\mathrm{KCO} 2$ & Materials Sciences and Engineering & 3,607 & 2,050 & 2,680 & 2,977 \\
\hline \multicolumn{2}{|r|}{ Total Accelerator Improvement Projects } & 3,607 & 2,050 & 2,680 & 2,977 \\
\hline \multicolumn{6}{|c|}{ GENERAL PLANT PROJECTS: } \\
\hline FS10 & Safeguards and Security-Science & 9 & (9) & - & - \\
\hline KA11 & Proton Accelerator-Based Physics (b) & 3,248 & 4,188 & 4,340 & 3,095 \\
\hline $\mathrm{KCO} 2$ & Materials Sciences and Engineering & - & 600 & - & 600 \\
\hline KG09 & General Plant Projects & 0 & $=$ & 0 & 0 \\
\hline KP11 & Life Sciences & 67 & - & - & 67 \\
\hline $\mathrm{KP} 13$ & Environmental Remediation & 4 & (4) & - & - \\
\hline \multicolumn{2}{|r|}{ Total General Plant Projects } & 3,328 & 4,775 & 4,340 & 3,763 \\
\hline \multicolumn{6}{|c|}{ LINE ITEM CONSTRUCTION: } \\
\hline $39 \mathrm{KC}$ & Basic Energy Sciences & 1.101 & 4.954 & 2,495 & 3,560 \\
\hline $39 K G$ & Science Laboratories Infrastructure & 16,491 & 9.272 & 6,066 & 19,697 \\
\hline \multicolumn{2}{|r|}{ Total Line Item Construction } & 17,592 & 14,226 & 8,561 & 23,256 \\
\hline \multicolumn{2}{|c|}{ Total Office of Science } & 169,293 & 451,248 & 424,905 & 195,636 \\
\hline \multicolumn{6}{|c|}{$\begin{array}{l}\text { Note: Minor variances may occur due to rounding. Sub-program titles may have changed since publication of the } \\
\text { FY2007 Annual Report. }\end{array}$} \\
\hline
\end{tabular}


Table 2.4

DOE Programs (\$K) Continued

\begin{tabular}{|c|c|c|c|c|c|}
\hline \multicolumn{2}{|c|}{ Assistant Secretary for Energy Efficiency and Renewable Energy } & $\begin{array}{c}\text { FY2008 } \\
\text { Beginning } \\
\text { Uncosted } \\
\text { Obligations }\end{array}$ & $\begin{array}{l}\text { FY2008 } \\
\text { Funds }\end{array}$ & $\begin{array}{l}\text { FY2008 } \\
\text { Costs }\end{array}$ & $\begin{array}{l}\text { FY2008 } \\
\text { Ending } \\
\text { Uncosted } \\
\text { Obligations }\end{array}$ \\
\hline \multicolumn{6}{|c|}{ OPERATING: } \\
\hline BMO1 & Biomass/Biofuels Energy Systems & + & 478 & - & 478 \\
\hline BTOI & Residential Buildings & 143 & 960 & 578 & 525 \\
\hline BT02 & Commercial Buildings Integration & 903 & 1,371 & 997 & 1.277 \\
\hline BTO3 & Emerging Technologies & 1,072 & 2,745 & 3.295 & 522 \\
\hline BTO4 & Equipment Standards and Analysis & 1,061 & 2,800 & 2,858 & 1,004 \\
\hline BT05 & Technical Program Management Support & 15 & - & 7 & 9 \\
\hline BT07 & Technology Validation and Market Distribution & 97 & 105 & 165 & 38 \\
\hline EB21 & Solar Energy & 77 & 164 & 108 & 132 \\
\hline EB25 & Wind Energy Systems & 308 & 945 & 508 & 745 \\
\hline EB 40 & Geothermal & 617 & 1,341 & 783 & 1,175 \\
\hline $\mathrm{EB} 42$ & Hydrogen Research R\&D & 111 & 2,593 & 1,560 & 1,144 \\
\hline EB55 & Department Energy Management Program & 209 & - & (6) & 215 \\
\hline EB57 & $\begin{array}{l}\text { Energy Efficiency and Renewable Energy (EERE) Program } \\
\text { Support }\end{array}$ & 130 & 90 & 95 & 124 \\
\hline ED18 & Industries Of The Future (Specific) & 12 & - & 8 & 4 \\
\hline ED19 & Industries Of The Future (Cross-cutting) & 1,409 & 2,347 & 2,155 & 1,601 \\
\hline ED22 & Technical Program Management Support & 38 & - & 2 & 36 \\
\hline EHOI & Program Direction - CRE & 0 & (0) & - & - \\
\hline $\mathrm{EH} 25$ & Planning, Evaluation and Analysis & 7 & - & 4 & 3 \\
\hline EL 17 & Federal Energy Management Program & 1,302 & 2,305 & 1.727 & 1,880 \\
\hline EOO1 & Distributed Energy Resources & 0 & (0) & - & 0 \\
\hline $\mathrm{H} 103$ & Stack Component R\&D & 4 & - & 4 & 0 \\
\hline VTO3 & Hybrid and Electric Propulsion & 3,072 & - & 3,017 & 55 \\
\hline VT05 & Materials Technology & 50 & - & 49 & 0 \\
\hline$V \pi 11$ & Hybrid Electric Systems & - & 8,170 & 4,857 & 3,313 \\
\hline W101 & Intergovernmental Activities & 23 & - & 11 & 12 \\
\hline W103 & State Energy Program (Grants) & - & 111 & - & 111 \\
\hline W104 & Other State Energy Activities & 1,363 & 263 & 624 & 1,001 \\
\hline W105 & Gateway Deployment & 17 & 114 & 16 & 116 \\
\hline W106 & Intergovernmental Activities & 2,497 & 200 & 636 & 2,061 \\
\hline W107 & Weatherization Assistance Program & 24 & - & - & 24 \\
\hline \multicolumn{2}{|r|}{ Total Operating } & 14,562 & 27,102 & 24,059 & 17,604 \\
\hline
\end{tabular}

continued... 
Table 2.4

\section{DOE Programs (\$K) Continued}

\begin{tabular}{|c|c|c|c|c|c|}
\hline \multicolumn{2}{|c|}{$\begin{array}{l}\text { Assistant Secretary for Energy Efficiency and Renewable Energy } \\
\text { (continued) }\end{array}$} & $\begin{array}{c}\text { FY2008 } \\
\text { Beginning } \\
\text { Uncosted } \\
\text { Obligations }\end{array}$ & $\begin{array}{c}\text { FY2008 } \\
\text { Funds }\end{array}$ & $\begin{array}{l}\text { FY2008 } \\
\text { Costs }\end{array}$ & $\begin{array}{l}\text { FY2008 } \\
\text { Ending } \\
\text { Uncosted } \\
\text { Obligations }\end{array}$ \\
\hline \multicolumn{6}{|c|}{ CAPITAL EQUIPMENT: } \\
\hline BTO3 & Emerging Technologies & 192 & - & 99 & 94 \\
\hline EB42 & Hydrogen Research R\&D & - & 100 & - & 100 \\
\hline VTO3 & Hybrid and Electric Propulsion & 651 & - & 586 & 65 \\
\hline VT05 & Materials Technology & 3 & (3) & - & - \\
\hline $\mathrm{V} T 11$ & Hybrid Electric Systems & $=$ & 1,000 & 78 & 922 \\
\hline \multicolumn{2}{|r|}{ Total Capital Equipment } & 846 & 1,097 & 763 & 1,180 \\
\hline \multicolumn{2}{|c|}{ Total Assistant Secretary for Energy Efficiency and Renewable Energy } & 15,408 & 28,199 & 24,822 & 18,785 \\
\hline
\end{tabular}

\begin{tabular}{|c|c|c|c|c|c|}
\hline \multicolumn{2}{|c|}{ Office of Electricity Delivery and Energy Reliability } & $\begin{array}{c}\text { FY2008 } \\
\text { Beginning } \\
\text { Uncosted } \\
\text { Obligations }\end{array}$ & $\begin{array}{l}\text { FY2008 } \\
\text { Funds }\end{array}$ & $\begin{array}{c}\text { FY2008 } \\
\text { Costs }\end{array}$ & $\begin{array}{c}\text { FY2008 } \\
\text { Ending } \\
\text { Uncosted } \\
\text { Obligations }\end{array}$ \\
\hline \multicolumn{6}{|c|}{ OPERATING: } \\
\hline TD50 & Research and Development & 959 & 1.935 & 1.971 & 922 \\
\hline TD52 & Electricity Restructuring & 206 & - & 205 & 0 \\
\hline TD54 & Operations and Analysis & 4,005 & 3,035 & 2,065 & 4,974 \\
\hline \multicolumn{2}{|r|}{ Total Operating } & 5,169 & 4,970 & 4,242 & 5,897 \\
\hline \multicolumn{2}{|c|}{ Total Office of Electricity Delivery and Energy Reliability } & 5,169 & 4,970 & 4,242 & 5,897 \\
\hline
\end{tabular}

continued... 
Table 2.4

\section{DOE Programs (\$K) Continued}

\begin{tabular}{|c|c|c|c|c|c|}
\hline \multicolumn{2}{|c|}{ Assistant Secretary for Fossil Energy } & $\begin{array}{c}\text { FY2008 } \\
\text { Beginning } \\
\text { Uncosted } \\
\text { Obligations }\end{array}$ & $\begin{array}{l}\text { FY2008 } \\
\text { Funds }\end{array}$ & $\begin{array}{l}\text { FY2008 } \\
\text { Costs }\end{array}$ & $\begin{array}{l}\text { FY2008 } \\
\text { Ending } \\
\text { Uncosted } \\
\text { Obligations }\end{array}$ \\
\hline \multicolumn{6}{|c|}{ OPERATING: } \\
\hline AA15 & Advanced Research & 163 & (3) & 124 & 35 \\
\hline $\mathrm{AA} 20$ & Central Systems & 797 & 696 & 742 & 751 \\
\hline $\mathrm{AA} 25$ & Fuel Cells & 0 & 300 & 300 & 0 \\
\hline AA30 & Sequestration & 3,894 & 4.760 & 2,931 & 5,722 \\
\hline AB05 & Natural Gas Technologies & 728 & 918 & 1.231 & 415 \\
\hline$A C 10$ & Oil Technology & 2,184 & 423 & 930 & 1,677 \\
\hline $\mathrm{AD} 20$ & Contractual Services And Supplies & 200 & - & 194 & 6 \\
\hline AE 10 & Advanced Metallurgical Processes & 1 & - & - & 1 \\
\hline AK20 & Clean Coal Contractual Services and Supplies & 171 & - & 171 & - \\
\hline AY05 & Clean Coal Power Initiative & - & 30 & - & 30 \\
\hline CEO3 & Center for Zero Emissions Technology - Montana State & $=$ & 1,000 & 436 & 564 \\
\hline \multicolumn{2}{|r|}{ Total Operating } & 8,138 & 8,124 & 7,060 & 9,202 \\
\hline \multicolumn{2}{|c|}{ Total Assistant Secretary for Fossil Energy } & 8,138 & 8,124 & 7,060 & 9,202 \\
\hline \multicolumn{6}{|c|}{$\begin{array}{l}\text { Note: Minor variances may occur due to rounding. Sub-program titles may have changed since publication of the } \\
\text { FY2007 Annual Report. }\end{array}$} \\
\hline \multicolumn{2}{|c|}{ Office of Civilian Radioactive Waste Management } & $\begin{array}{c}\text { FY2008 } \\
\text { Beginning } \\
\text { Uncosted } \\
\text { Obligations }\end{array}$ & $\begin{array}{l}\text { FY2008 } \\
\text { Funds }\end{array}$ & $\begin{array}{c}\text { FY2008 } \\
\text { Costs }\end{array}$ & $\begin{array}{l}\text { FY2008 } \\
\text { Ending } \\
\text { Uncosfed } \\
\text { Obligations }\end{array}$ \\
\hline \multicolumn{6}{|c|}{ OPERATING: } \\
\hline DFO1 & First Repository & 171 & - & 104 & 67 \\
\hline DFO9 & Program Support & 2,182 & (0) & 1,975 & 207 \\
\hline & Total Operating & 2,353 & $(0)$ & 2,078 & 274 \\
\hline \multicolumn{2}{|c|}{ Total Office of Civilian Radioactive Waste Management } & 2,353 & (0) & 2,078 & 274 \\
\hline
\end{tabular}


DOE Programs (\$K) Continued

\begin{tabular}{|c|c|c|c|c|c|}
\hline \multicolumn{2}{|c|}{ Assistant Secretary for Environmental Management } & $\begin{array}{c}\text { FY2008 } \\
\text { Beginning } \\
\text { Uncosted } \\
\text { Obligations }\end{array}$ & $\begin{array}{l}\text { FY2008 } \\
\text { Funds }\end{array}$ & $\begin{array}{l}\text { FY2008 } \\
\text { Costs }\end{array}$ & $\begin{array}{l}\text { FY2008 } \\
\text { Ending } \\
\text { Uncosted } \\
\text { Obligations }\end{array}$ \\
\hline \multicolumn{6}{|c|}{ OPERATING: } \\
\hline EZO6 & $\begin{array}{l}\text { Non-Defense Site Acceleration Completion - } \\
2006 \text { Accelerated Completions }\end{array}$ & 0 & - & - & 0 \\
\hline EZ50 & Non-Defense Environmental Cleanup - Small Sites & 1,214 & - & 242 & 972 \\
\hline \multicolumn{2}{|r|}{ Total Operating } & 1,214 & - & 242 & 972 \\
\hline \multicolumn{2}{|c|}{ Total Assistant Secretary for Environmental Management } & 1,214 & - & 242 & 972 \\
\hline
\end{tabular}

\begin{tabular}{|l|c|c|c|c|}
\hline Office of Health Safety And Security & $\begin{array}{c}\text { FY2008 } \\
\text { Beginning } \\
\text { Uncosted } \\
\text { Obligations }\end{array}$ & $\begin{array}{c}\text { FY2008 } \\
\text { Funds }\end{array}$ & $\begin{array}{c}\text { FY2008 } \\
\text { Costs }\end{array}$ & $\begin{array}{c}\text { FY2008 } \\
\text { Unding } \\
\text { Uncosted } \\
\text { Obligations }\end{array}$ \\
\hline
\end{tabular}

\section{OPERATING:}

\begin{tabular}{|c|c|c|c|c|c|}
\hline HAIO & Worker Advocacy & 0 & $=$ & $=$ & 0 \\
\hline HD10 & Other Defense Activities - Operating & 0 & - & - & 0 \\
\hline $\mathrm{HD} 20$ & Health & 0 & $=$ & $=$ & 0 \\
\hline HQ10 & Employee Compensation & 8 & 25 & 24 & 9 \\
\hline HU2O & Health & 275 & 388 & 518 & 145 \\
\hline & Total Operating & 283 & 413 & 542 & 154 \\
\hline \multicolumn{2}{|c|}{ Total Office of Health Safety And Security } & 283 & 413 & 542 & 154 \\
\hline
\end{tabular}




\section{DOE Programs (\$K) Continued}

\begin{tabular}{|c|c|c|c|c|c|}
\hline \multicolumn{2}{|c|}{ Assistant Secretary for Nuclear Energy } & $\begin{array}{c}\text { FY2008 } \\
\text { Beginning } \\
\text { Uncosted } \\
\text { Obligations }\end{array}$ & $\begin{array}{l}\text { FY2008 } \\
\text { Funds }\end{array}$ & $\begin{array}{l}\text { FY2008 } \\
\text { Costs }\end{array}$ & $\begin{array}{c}\text { FY2008 } \\
\text { Ending } \\
\text { Uncosted } \\
\text { Obligations }\end{array}$ \\
\hline \multicolumn{6}{|c|}{ OPERATING: } \\
\hline AF58 & $\begin{array}{l}\text { Advanced Fuel Cycle Initiative (AFCI)/Global Nuclear } \\
\text { Energy Partnership (GNEP) }\end{array}$ & 0 & 788 & 206 & 583 \\
\hline & Total Operating & - & 788 & 206 & 583 \\
\hline \multicolumn{2}{|c|}{ Total Assistant Secretary for Nuclear Energy } & - & 788 & 206 & 583 \\
\hline
\end{tabular}

\begin{tabular}{|c|c|c|c|c|c|}
\hline \multicolumn{2}{|c|}{ Office of Energy and Threat } & $\begin{array}{c}\text { FY2008 } \\
\text { Beginning } \\
\text { Uncosfed } \\
\text { Obligations }\end{array}$ & $\begin{array}{c}\text { FY2008 } \\
\text { Funds }\end{array}$ & $\begin{array}{l}\text { FY2008 } \\
\text { Costs }\end{array}$ & $\begin{array}{l}\text { FY2008 } \\
\text { Ending } \\
\text { Uncosted } \\
\text { Obligations }\end{array}$ \\
\hline \multicolumn{6}{|c|}{ OPERATING: } \\
\hline GD40 & Program Direction & - & 65 & - & 65 \\
\hline \multicolumn{2}{|r|}{ Total Operating } & - & 65 & - & 65 \\
\hline \multicolumn{2}{|c|}{ Total Office of Energy and Threat } & - & 65 & - & 65 \\
\hline
\end{tabular}

\begin{tabular}{|c|c|c|c|c|c|}
\hline \multicolumn{2}{|c|}{ Assistant Secretary for Policy and International Affairs } & $\begin{array}{l}\text { FY2008 } \\
\text { Beginning } \\
\text { Uncosted } \\
\text { Obligations }\end{array}$ & $\begin{array}{l}\text { FY2008 } \\
\text { Funds }\end{array}$ & $\begin{array}{c}\text { FY2008 } \\
\text { Costs }\end{array}$ & $\begin{array}{c}\text { FY2008 } \\
\text { Ending } \\
\text { Uncosted } \\
\text { Obligations }\end{array}$ \\
\hline \multicolumn{6}{|c|}{ OPERATING: } \\
\hline PEO1 & Policy, Planning, and Analysis & - & 65 & - & 65 \\
\hline \multicolumn{2}{|r|}{ Total Operating } & - & 65 & - & 65 \\
\hline \multicolumn{2}{|c|}{ Total Assistant Secretary for Policy and International Affairs } & - & 65 & - & 65 \\
\hline
\end{tabular}


Table 2.5

\section{Other Direct Operating (\$K)}

\begin{tabular}{|c|c|c|c|c|}
\hline Reimbursable Work for Other Agencies & $\begin{array}{c}\text { FY2008 } \\
\text { Beginning } \\
\text { Uncosted } \\
\text { Obligations }\end{array}$ & $\begin{array}{l}\text { FY2008 } \\
\text { Funds }\end{array}$ & $\begin{array}{l}\text { FY2008 } \\
\text { Costs }\end{array}$ & \begin{tabular}{|c|} 
FY2008 \\
Ending \\
Uncosted \\
Obligations \\
\end{tabular} \\
\hline \multicolumn{5}{|l|}{ Work for Other Federal Agencies } \\
\hline Department of Agriculture & 45 & - & 1 & 44 \\
\hline Department of Commerce & 70 & 10 & 31 & 50 \\
\hline Department of Defense & 14,973 & 7.472 & 7,977 & 14,621 \\
\hline Department of interior & 258 & 264 & 187 & 339 \\
\hline Department of Homeland Security - Borders \& Transportation & 83 & - & 83 & 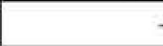 \\
\hline Department of Homeland Security - Domestic Nuclear Detection Office & - & 400 & 67 & 333 \\
\hline $\begin{array}{l}\text { Department of Homeland Security - Information Analysis and } \\
\text { Infrastructure Protection }\end{array}$ & 0 & - & $=$ & 0 \\
\hline Department of Homeland Security - Science \& Technology & 1,452 & 3,572 & 3,039 & 1.986 \\
\hline Department of State - International Affairs \& Energy Emergencies & 971 & 1.842 & 225 & 2,594 \\
\hline Environmental Protection Agency & 4,884 & 2,318 & 2,658 & 4.646 \\
\hline National Aeronqutics and Space Administration (NASA) & 4,219 & 3,204 & 4,927 & 2,653 \\
\hline National Institutes of Health & 51,234 & 40,836 & 43,110 & 48,958 \\
\hline National Science Foundation & 225 & 297 & 300 & 226 \\
\hline Nuclear Regulatory Commission & 0 & 199 & - & 199 \\
\hline Other Federal Agencies - Defense Related & 86 & 344 & 231 & 201 \\
\hline Other Federal Agencies - Energy Related & 1,090 & (449) & 196 & 451 \\
\hline Other Energy Related Activities & 826 & 1.332 & 1.164 & 1.011 \\
\hline Total Work for Other Fe deral Agencies & 80,418 & 61,640 & 64,195 & 78,315 \\
\hline \multicolumn{5}{|l|}{ Work for Non-Federal Agencies } \\
\hline Foreign Governments & 334 & 185 & 307 & 222 \\
\hline Industry & 5,644 & 12,748 & 12.290 & 6.423 \\
\hline State and Local Governments \& NPO's & 8,180 & 15,740 & 15.497 & 8,422 \\
\hline Universities and Institutes & 7.219 & 14,088 & 14,404 & 6.664 \\
\hline Cost of Work for Others Program (WN) (a) & 3,006 & 1.121 & 914 & 3,217 \\
\hline Total Work for Non-Federal Agencies & 24,383 & 43,882 & 43,412 & 24,947 \\
\hline \multicolumn{5}{|l|}{ Cooperative Research and Development Agreements } \\
\hline CRADA - Small Business & 83 & 99 & 117 & 65 \\
\hline CRADA - Other & 152 & 440 & 335 & 270 \\
\hline Total Cooperative Rese arch and Development Agreements & 235 & 539 & 452 & 335 \\
\hline Total Reimbursable Work for Others & 105,037 & 106,061 & 108,059 & 103,597 \\
\hline
\end{tabular}


Table 2.5

\section{Other Direct Operating (\$K) Continued}

\begin{tabular}{|c|c|c|c|c|}
\hline Work for Other DOE Integrated Contractors & $\begin{array}{c}\text { FY2008 } \\
\text { Beginning } \\
\text { Uncosted } \\
\text { Obligations }\end{array}$ & $\begin{array}{l}\text { FY2008 } \\
\text { Funds }\end{array}$ & $\begin{array}{c}\text { FY2008 } \\
\text { Costs }\end{array}$ & $\begin{array}{c}\text { FY2008 } \\
\text { Ending } \\
\text { Uncosted } \\
\text { Obligations } \\
\end{array}$ \\
\hline Work Performed for Other DOE Locations (b) & - & 10,296 & 10,296 & - \\
\hline Total Work for Other DOE Inte grated Contractors & - & 10,296 & 10,296 & - \\
\hline Total Other Direct Operating (c) & 105,037 & 116,357 & 118,355 & 103,597 \\
\hline
\end{tabular}

Note: Minor variances may occur due to rounding.

(a) Includes funding for Non-Federal Sponsors who are precluded by law from paying an advance under the WNO2 program.

(b) Total funding for Work for Other Integrated Contractors is assumed to be equal to cost incurred.

(c) The sum of FY2008 Beginning Uncosted Obligations, FY2008 Funds, and FY2008 Costs does not equal FY2008 Ending Uncosted Obligations due to various adjustments not reflected in the FY2008 Costs column. Examples of these adjustments include bridge funding, suspense items, and the Federal Administrative Charge. The total of these adjustments for FY2008 is ( $\$ 558 \mathrm{~K}$ ). 
Figure 2.1

\section{Sponsored Project Information (\$K)}

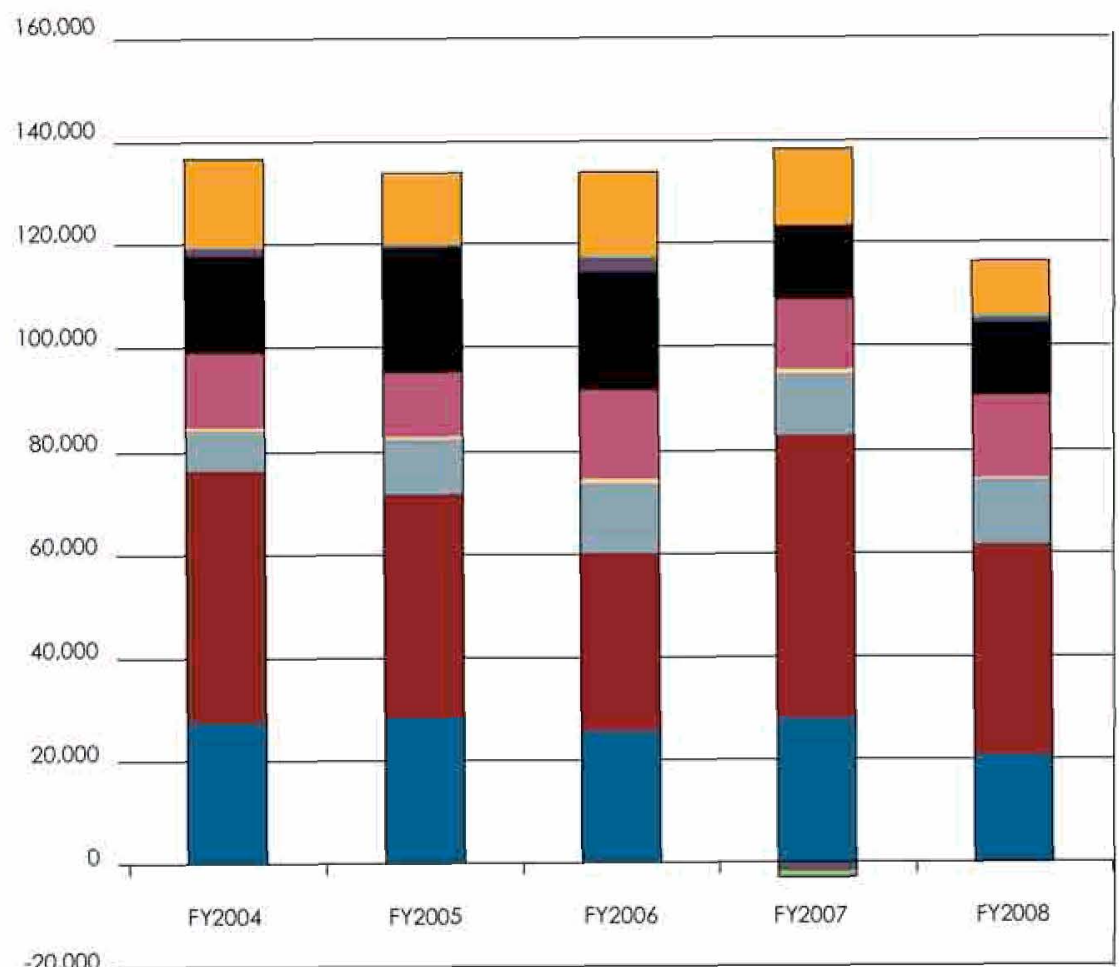

Reimbursable

Work-for-Others

Annual Funding

Information (\$K)
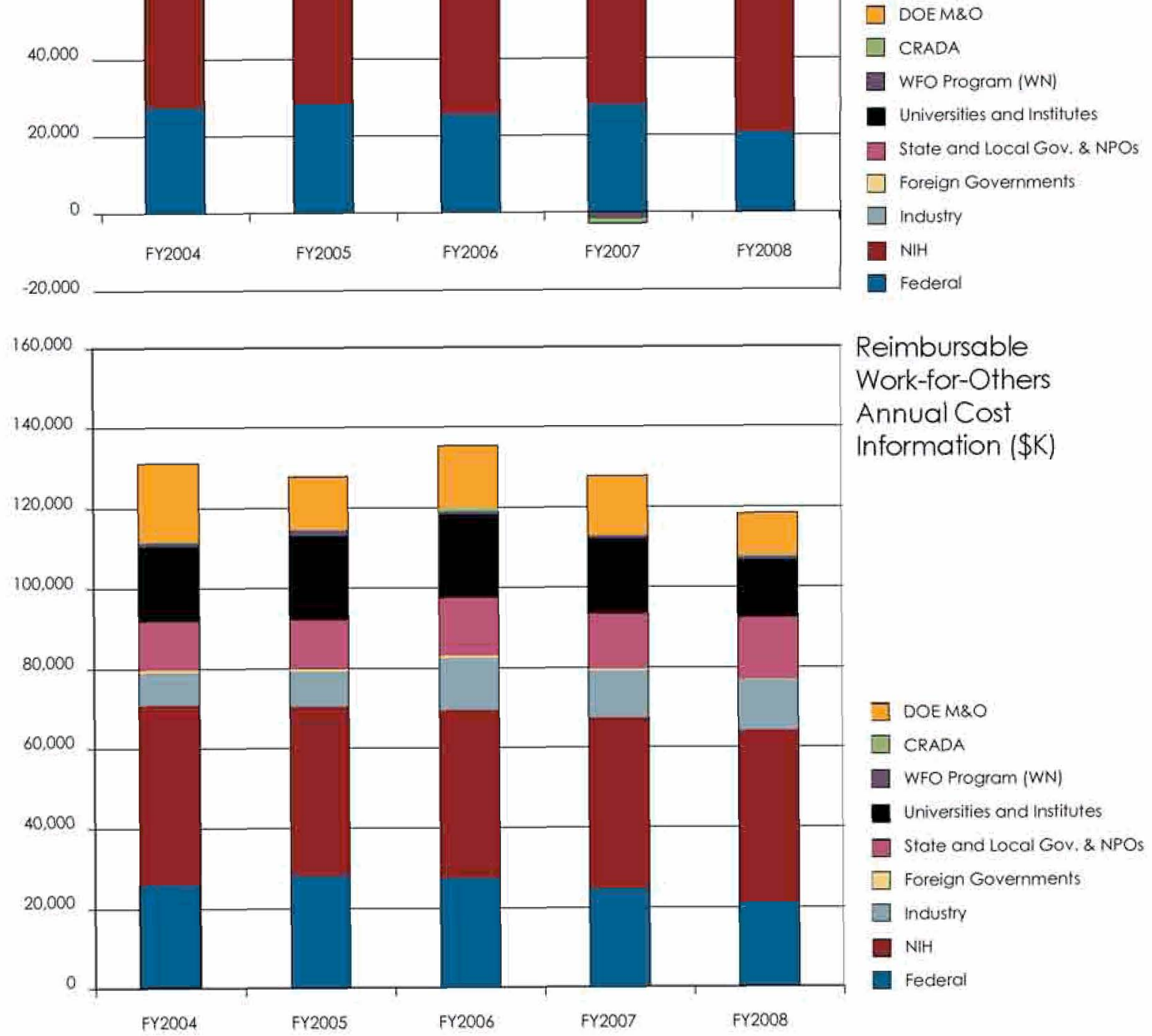
3. Indirect Budgets 
Figure 3.1

\section{Indirect Budgets - FY2008 Costs (\$M)}

\begin{tabular}{|l|r|}
\hline Indirect Budgets (a) & FY2008 Costs (\$M) \\
\hline G\&A (Includes Site Support) & 113.5 \\
\hline Organization Burden & 38.1 \\
\hline Service Centers (b) & 22.9 \\
\hline LDRD & 18.3 \\
\hline IGPP & 2.5 \\
\hline Procurement & 9.9 \\
\hline Other (c) & 0.2 \\
\hline Travel & \\
\hline Total & \\
\hline
\end{tabular}

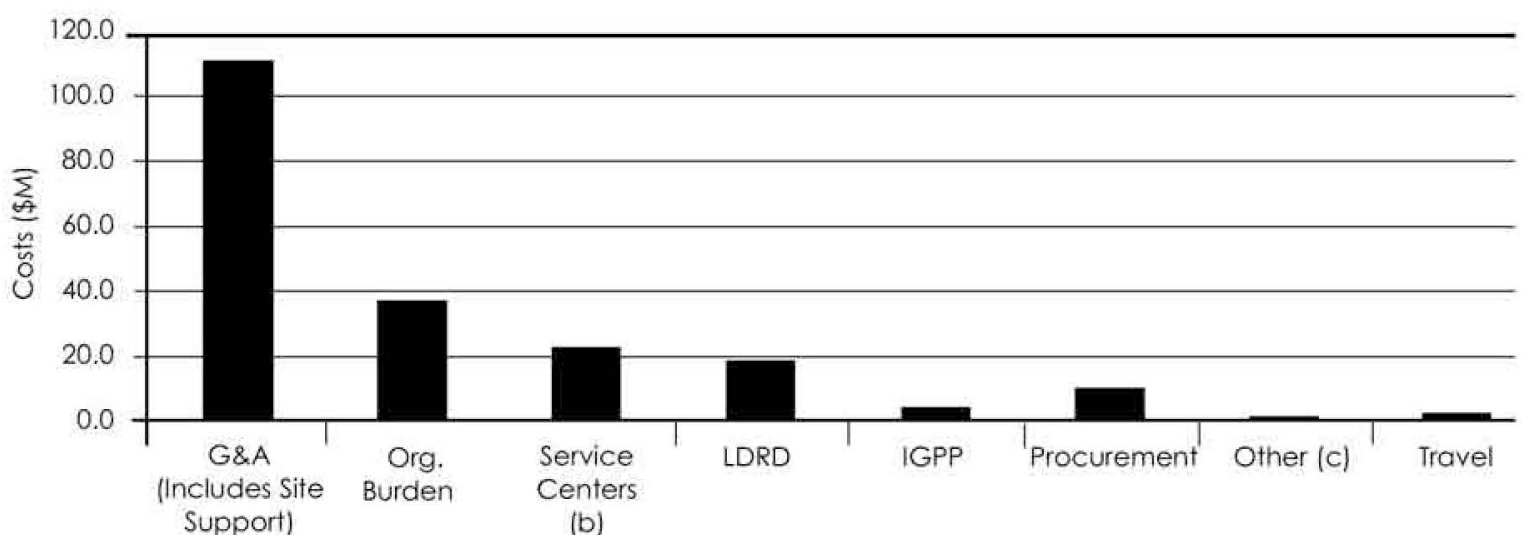

(a) Summation of indirect budget provided only to show magnitude of dollars being managed and does not equate to total indirect costs since there are overlaps between indirect budgets. For example, some organization burden costs are included in G\&A and Recharges. In FY2008 LDRD cost includes \$4.9M G\&A assessed on LDRD projects.

(b) Service Centers includes recharge cost centers that default to B\&R YNO1 (project type OHRCH) only.

(c) Includes Office of Homeland Security Charge. 


\section{Institutional Overhead Costs as a Percent of Operating Costs, FY2006 - FY2008}

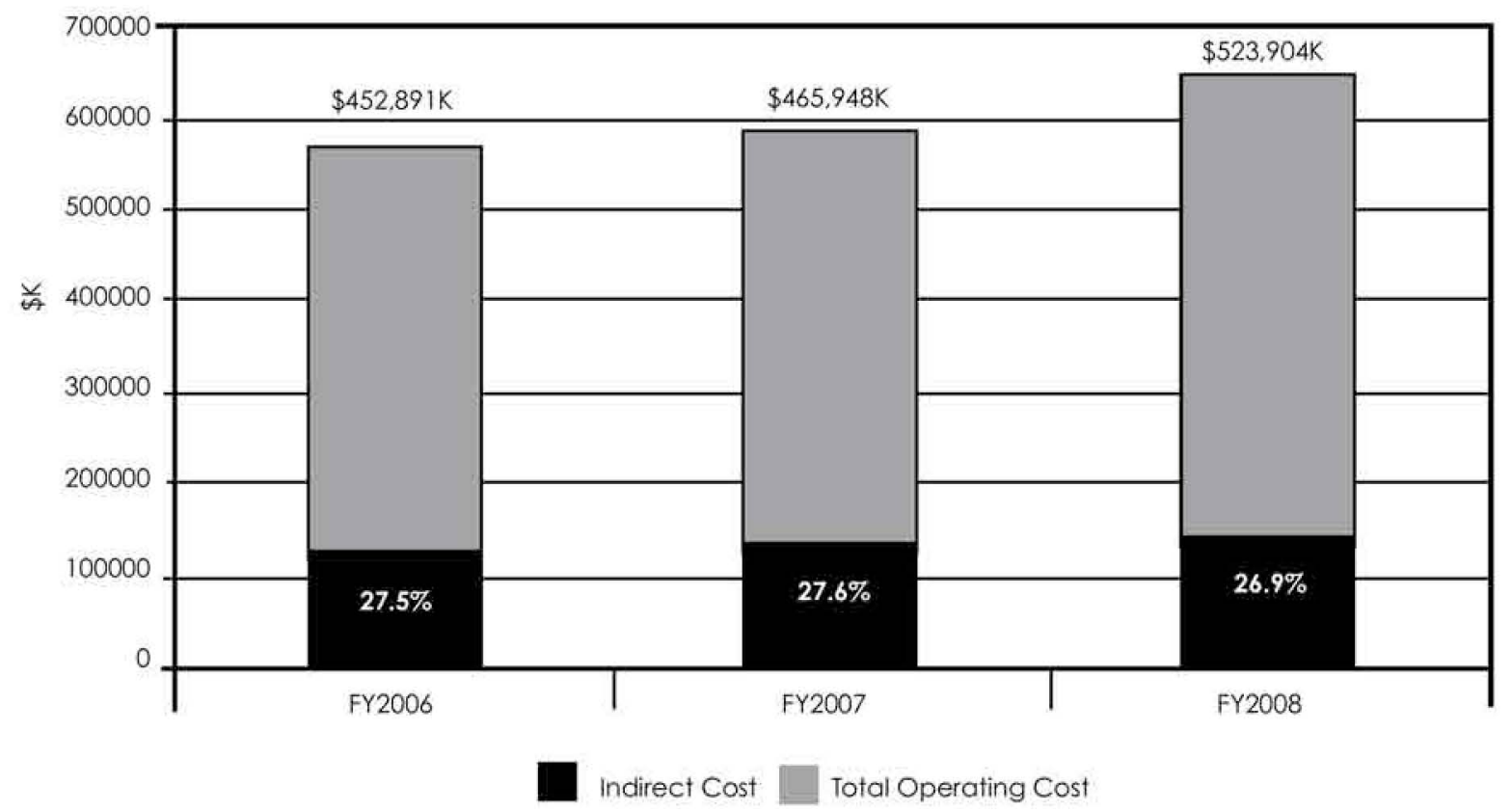

Note: Chart represents the institutional overhead costs structure for each fiscal year with adjustments for indirect double count of G\&A on LDRD projects (DOE mandate to apply G\&A to LDRD projects beginning FY2006). Institutional overhecd costs include G\&A, Site Support, LDRD, IGPP, Procurement, and Travel. Percent is the percentage of indirect cost to total operating cost. 
Table 3.1

\section{Institutional Costs by Division, FY2008 (\$K)}

\begin{tabular}{|c|c|c|c|c|c|c|}
\hline Division & $G \& A(a)$ & LDRD (b) & Procurement & Travel & IGPP & Total \\
\hline LABORATORY DIRECTORATE & 10,911 & & & & & 10,911 \\
\hline LDRD & & 18,252 & & & & 18,252 \\
\hline ENGINEERING & 2,406 & & & & & 2,406 \\
\hline \multicolumn{7}{|l|}{ ALD FOR OPERATIONS } \\
\hline ALD Office & 978 & & & & & 978 \\
\hline IGPP & & & & & 2,452 & 2,452 \\
\hline Non-Cap & 4,511 & & & & & 4,511 \\
\hline Work Force Diversity Office & 445 & & & & & 445 \\
\hline Public Affairs & 1,973 & & & & & 1,973 \\
\hline$H R$ & 4,550 & & & & & 4,550 \\
\hline EH\&S & 20,199 & & & & & 20,199 \\
\hline Facilities & 33,240 & & 2,163 & & & 35,403 \\
\hline CFO & 7.405 & & 7,584 & 1,408 & & 16,398 \\
\hline IT & 20,817 & & 180 & & & 20,997 \\
\hline GENERAL LABORATORY & 6,030 & & & & & 6,030 \\
\hline Total & 113,464 & 18,252 & 9,928 & 1,408 & 2,452 & 145,504 \\
\hline
\end{tabular}


Table 3.2

\section{Institutional FTEs Charged by Division, FY2008}

\begin{tabular}{|c|c|c|c|c|c|c|}
\hline Division & G\&A (a) & LDRD (b) & Procurement & Travel & IGPP & Total \\
\hline LABORATORY DIRECTORATE & 54.3 & & & & & 5.3 \\
\hline LDRD (b) & & 78.6 & & & & 78.6 \\
\hline ENGINEERING & 8.0 & & & & & 8.0 \\
\hline \multicolumn{7}{|l|}{ ALD FOR OPERATIONS } \\
\hline ALD Office & 5.5 & & & & & 5.5 \\
\hline IGPP & & & & & 3.5 & 3.5 \\
\hline Non-Cap & 7.6 & & & & & 7.6 \\
\hline Work Force Diversity Office & 3.1 & & & & & 3.1 \\
\hline Public Affairs & 12.6 & & & & & 12.6 \\
\hline$H R$ & 34.9 & & & & & 34.9 \\
\hline EH\&S & 103.8 & & & & & 103.8 \\
\hline Facilities & 124.1 & & 22.4 & & & 146.5 \\
\hline OCFO & 62.0 & & 59.8 & 9.3 & & 131.1 \\
\hline IT & 90.5 & & 1.1 & & & 91.6 \\
\hline GENERAL LABORATORY & 0.1 & & & & & 0.1 \\
\hline Total & 506.4 & 78.6 & 83.3 & 9.3 & 3.5 & 681.1 \\
\hline $\begin{array}{l}\text { Note: Minor variances may od } \\
\text { (a) Includes Site Support \& Str } \\
\text { (b) LDRD projects conducted }\end{array}$ & $\begin{array}{l}\text { due to rour } \\
\text { ic Planning } \\
\text { nultiple divi }\end{array}$ & pport Acti & $\begin{array}{l}\text { (SPSA). } \\
\text { in Table } 1.3 .\end{array}$ & & & \\
\hline
\end{tabular}




\section{Payroll Burden Summary ( $\$ M)$}

Figure 3.3

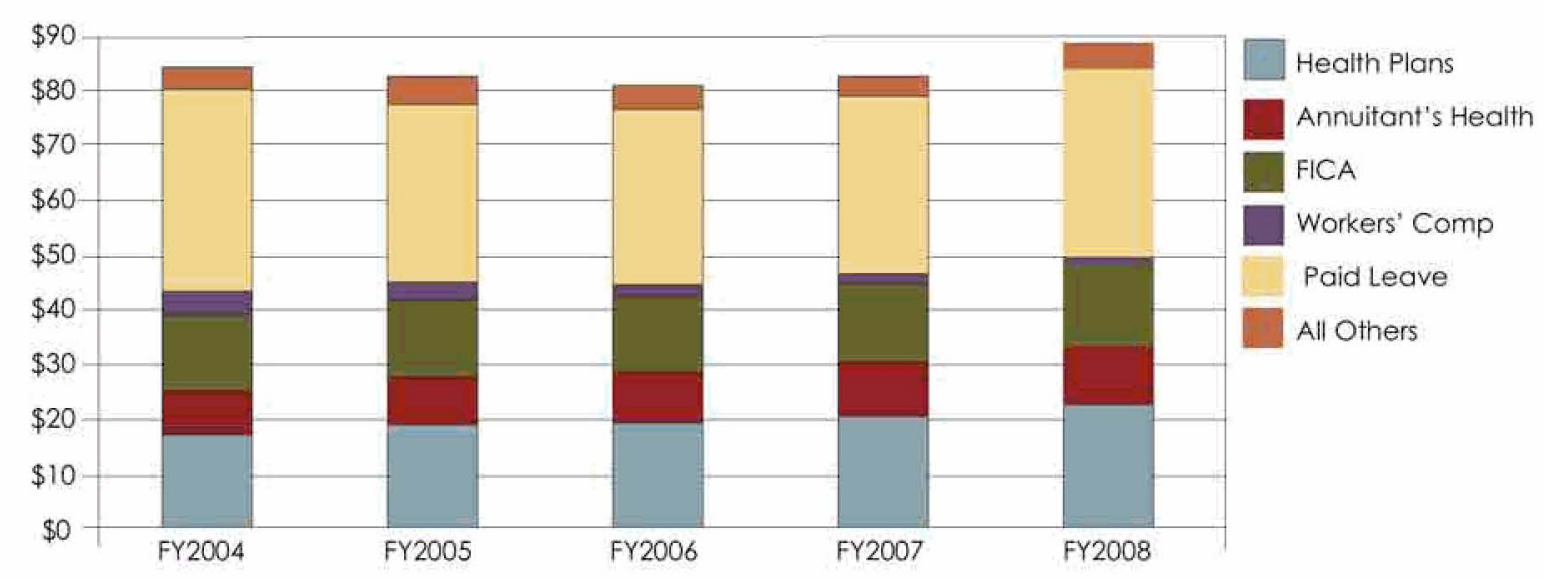

Figure 3.4

\section{Gross Payroll Summary (\$M)}

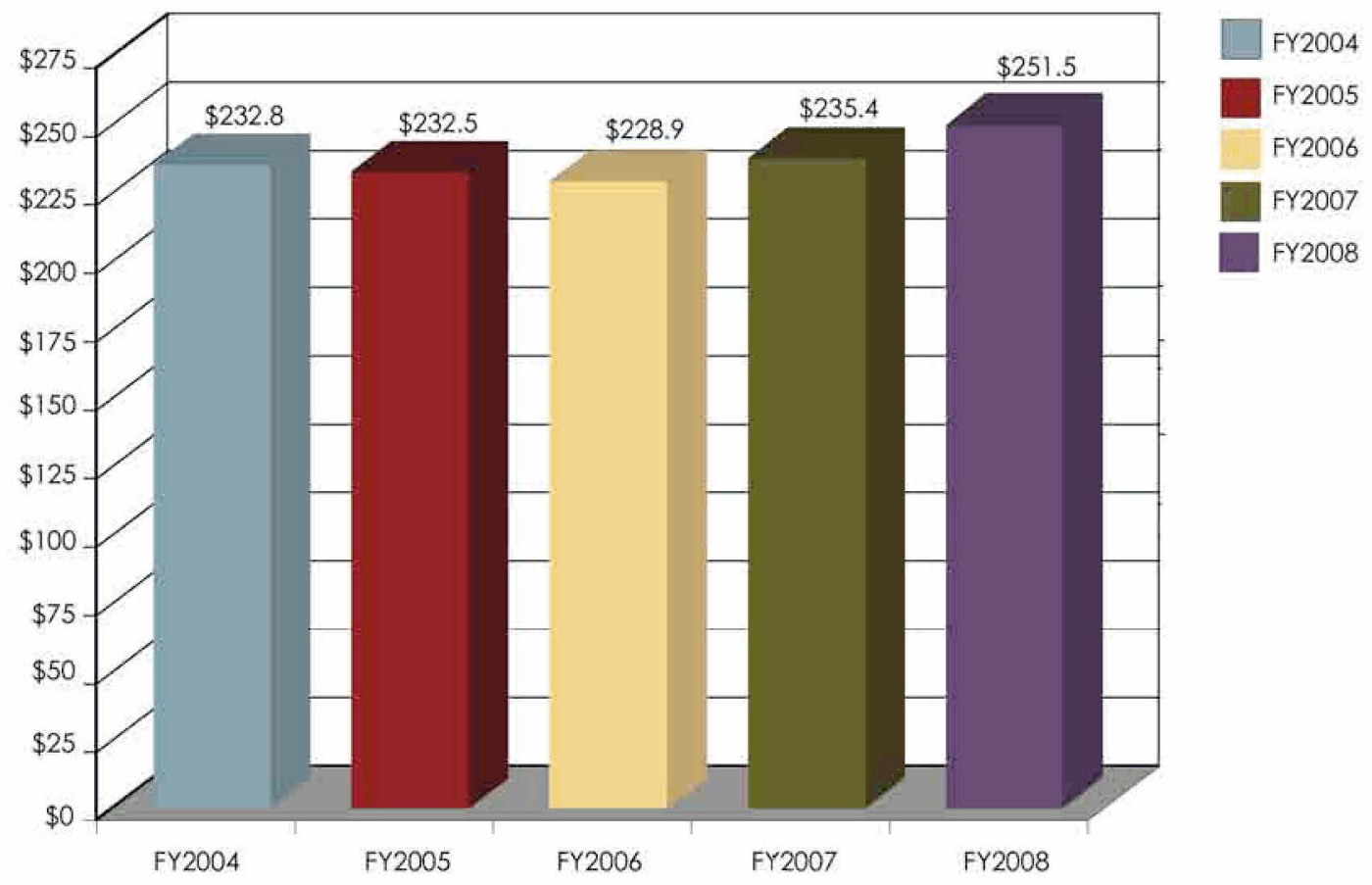

Fiscal Year 
Table 3.3

\section{Organizational Burden Costs and FTEs}

Organizational burden includes costs for the management and supervision of division/department activities and is distributed over labor costs including campus and contract labor.

\begin{tabular}{|l|r|r|}
\hline \multirow{2}{*}{ Division Cost Pools } & \multicolumn{2}{|c|}{ FY2008 } \\
\cline { 2 - 3 } & \multicolumn{1}{|c|}{ Cost \$K FTE } \\
\hline Accelerator \& Fusion Research & 1,553 & 10.8 \\
\hline Advanced Light Source & 1,911 & 13.1 \\
\hline Chemical Sciences & 952 & 6.6 \\
\hline Computing Sciences & 4,513 & 33.8 \\
\hline Environmental Energy Technology & 3,200 & 24.4 \\
\hline Engineering & 4,701 & 30.1 \\
\hline Earth Sciences & 2,646 & \\
\hline Facilities & 3,368 & \\
\hline Genomics - Onsite & 480 & \\
\hline Information Technology & 21.7 \\
\hline Life Sciences & 2,795 & \\
\hline Materials Sciences & 4,390 & \\
\hline Nuclear Sciences & 4.6 \\
\hline Physical Biosciences & 15.1 \\
\hline Physics & 3.942 & \\
\hline Total & 1,336 & \\
\hline Note: Minor Variances may occur due to rounding. & 1,982 & \\
\hline
\end{tabular}

Table 3.4

\section{Service Center Costs and FTEs}

Certain Laboratory services are provided by recharges that recover operational costs through various cost-allocation mechanisms; e.g. by assigning a dollar value to the work performed (a unit charge based on an hourly rate) or the products produced (unit charge per item).

\begin{tabular}{|l|r|r|}
\hline \multirow{2}{*}{ Division (a) } & \multicolumn{2}{|c|}{ FY2008 } \\
\cline { 2 - 3 } & \multicolumn{1}{|c|}{ Cost \$K } & 146 \\
\hline Accelerator \& Fusion Research & 193 & 0.4 \\
\hline Advanced Light Source Apartments & 1,456 & 0.3 \\
\hline Engineering & 1,113 & 8.0 \\
\hline Environmental Energy Technology & 9,575 & 9.3 \\
\hline Facilities & 6.777 & \\
\hline Information Technology & 4.3 \\
\hline Life Sciences & 499 \\
\hline Materials Sciences & 272 \\
\hline Physical Biosciences & 1,557 \\
\hline ALD Operations & 1,294 \\
\hline Total & 3.5 \\
\hline $\begin{array}{l}\text { Note: Minor Variances may occur due to rounding. } \\
\text { la) Service Centers includes recharge cost centers that default to B\&R YNOI (project type OHRCH) only. }\end{array}$ \\
\hline
\end{tabular}


Table 3.5

\section{Distributed Recharges by Resource Category Trends, FY2004 - FY2008 (\$K)}

\begin{tabular}{|c|c|c|c|c|c|}
\hline Distributed Recharge $(a, b)$ & FY2004 & FY2005 & FY2006 & FY2007 & FY2008 \\
\hline Vehicle & 1,285 & 1,267 & 1,498 & 1,190 & 1,204 \\
\hline MSD Facility & 540 & 473 & 367 & 316 & 273 \\
\hline Building Manager & 115 & 127 & 84 & 151 & 160 \\
\hline ALS Apartment Recharge (c) & & & 218 & 174 & 177 \\
\hline Animal Care & 537 & 446 & 444 & 408 & 396 \\
\hline Creative Services & 2,139 & 1.547 & 1,368 & 1.357 & 1.319 \\
\hline 88-Inch Accelerator Operations & 212 & 67 & 135 & 421 & 545 \\
\hline JBEI Non-Material Recharge & & & & & 70 \\
\hline JBEl Material Recharge & & & & & 1,487 \\
\hline Telephone Services & 6,909 & 6.222 & 4.753 & 4,080 & 3,980 \\
\hline EETD Recharge & & 1.095 & 1.077 & 1.065 & 1.106 \\
\hline Molecular Foundry & & 44 & 93 & 138 & 171 \\
\hline Computer/Net Recharges & 4,312 & 4,558 & 5,613 & 4,376 & 2,733 \\
\hline Engineering Shop (d) & 1.165 & 956 & 890 & 839 & 951 \\
\hline$C A D$ & 780 & 653 & 634 & 636 & 647 \\
\hline ALS Proprietary Recharge & 433 & 529 & 731 & 693 & 776 \\
\hline HTA Non-Material Recharge & & 5 & 33 & 38 & 49 \\
\hline HTA Material Recharge & & 42 & 153 & 60 & 53 \\
\hline JGI Recharge (Capillary Sequencing) & & 17,760 & 13,083 & 12,316 & 10,838 \\
\hline JGI Recharge (Synthesis Sequencing) & & & & 34 & 49 \\
\hline JGI WFO Administrative Charge & & 222 & 195 & 102 & 183 \\
\hline ESnet Recharge & 4,214 & 2,442 & 4.719 & 3,460 & 3,307 \\
\hline Electricity (e) & 8.153 & 8,072 & 6,335 & 7.307 & 8,382 \\
\hline Conference Recharge & 111 & 51 & 73 & 60 & \\
\hline Low Background Facility & 49 & 13 & 11 & 31 & 67 \\
\hline Print Room & 52 & 39 & 1 & & \\
\hline Biomed Isotopes & 189 & 141 & 91 & 51 & \\
\hline Mixed Waste Recharge/GL & & & 16 & 6 & 3 \\
\hline Miscellaneous Recharges & & & 39 & (0) & \\
\hline Rapid Prototyping Lab & (10) & 13 & & & \\
\hline \multicolumn{6}{|l|}{ DNA Sequencing } \\
\hline Total Recharges & 31,185 & 46,784 & 42,652 & 39,308 & 38,927 \\
\hline \multicolumn{6}{|c|}{$\begin{array}{l}\text { Note: Minor variances may occur due to rounding. } \\
\text { (a) Includes recharges credited back to direct operating accounts such as ALS, Esnet, JGI, etc. } \\
\text { (b) Does not include Procurement and Travel recharges. } \\
\text { (c) Prior to FY2006 recharge was incorporated within UCDRD funds. } \\
\text { (d) Prior to FY2003, CAD charges are included in Engineering Shop. } \\
\text { (e) Prior to FY2006 recharge included Electricity Maintenance, which moved to Site Support in FY2006. }\end{array}$} \\
\hline
\end{tabular}




\section{W 4. Financial Statement}




\section{Balance Sheet Comparative Statement of Financial Position (in \$K)}

\begin{tabular}{|c|c|c|}
\hline & FY2007 & FY2008 \\
\hline \multicolumn{2}{|l|}{ ASSETS: } & \\
\hline \multicolumn{3}{|l|}{ Current Assets } \\
\hline Accounts Receivable (Note 2) & 10,839 & 6,901 \\
\hline Inventories (Note 3) & 588 & 499 \\
\hline Other Current Assets (Note 4) & 1,167 & 516 \\
\hline Total Current Assets & 12,594 & 7,916 \\
\hline Pension Plan Assets & 536,627 & 442,432 \\
\hline Net Plant and Equipment (Note 5) & 577,747 & 589,046 \\
\hline Total Assets & $\$ 1,126,968$ & $1,039,394$ \\
\hline \multicolumn{3}{|l|}{ LIABILITIES AND EQUITY: } \\
\hline \multicolumn{3}{|l|}{ Liabilities: } \\
\hline \multicolumn{3}{|l|}{ Cument Liabilities } \\
\hline Drafts Payable (Note 6) & 1.017 & (976) \\
\hline Accounts Payable & 42,740 & 57,025 \\
\hline Accrued Expenses & 39,482 & 39,910 \\
\hline Other & 21.979 & 23,617 \\
\hline Total Current Liabilities & 108,218 & 119,576 \\
\hline Post-Retirement Benefits & 353,355 & 339,379 \\
\hline Environmental Liabilities (Note 7) & 778,716 & 613,076 \\
\hline Capital Lease Liability (Note 8) & & 18,946 \\
\hline ES\&H Liability (Note 9) & 145,575 & 458,428 \\
\hline Total Liabilities & $1,385,864$ & $1,549,405$ \\
\hline \multicolumn{3}{|l|}{ DOE Equity: } \\
\hline Beginning Equity & $(158,399)$ & $(258,896)$ \\
\hline Change in Equity & $(100,497)$ & $(251,115)$ \\
\hline Ending Equity & $(258,896)$ & $(510,011)$ \\
\hline Total Liabilities and Equity & $\$ 1,126,968$ & $1,039,394$ \\
\hline
\end{tabular}


Note 1

\section{Summary of Significant Accounting Policies}

\section{Basis of Presentation}

These financial statements have been prepared to report the financial position and results of operations of LBNL. They have been prepared from the books and records of the Laboratory in accordance with LBNL's accounting policies which are summarized in this note.

\section{Reporting Entity}

The Laboratory is a national research facility operated by UC for DOE under the terms of Contract DE-ACO205CH11231 (Contract 31). The Laboratory's reporting entity status is that of an integrated contractor, meaning LBNL's accounts are integrated with those of DOE through the use of reciprocal accounts. All of the assets and liabitities are owned by the Federal Government.

\section{Basis of Accounting}

The financial records of the Laboratory conform with generally accepted accounting principles (GAAP) and cost accounting standards (CAS) when they do not conflict with the provisions of the DOE accounting directives for Management and Operating (M\&O) Contractors and are in compliance with Contract 31 between UC and DOE.

\section{Financial Sources}

The Laboratory receives funding from DOE in accordance with the provisions of Contract 31. The Laboratory receives authorizations to incur costs and conduct operations through modifications to the contract.

Reimbursable work is performed for Federal and nonFederal entities. Costs are recorded and billed to the requesting entity by the Laboratory on behalf of DOE. Cash collected from these billings is transmitted to the U.S. Department of the Treasury and deposited in the DOE account. Non-Federally funded work performed at LBNL must be funded in advance.

\section{Letter of Credit}

The Laboratory received authority for expenditures according to a checks-paid letter of credit from the U.S. Department of the Treasury; Letter of Credit Contract Number DE-AC02-05CH1 1231 with Wells Fargo Bank effective April 1, 2007 to March 31, 2010, with two option years for possible extension to March 31, 2012.

\section{Inventories}

The Laboratory uses a perpetual inventory system for all inventories. An annual physical inventory is performed according to a cyclical sampling plan approved by DOE. Stores inventories and precious metals are valued and charged based on a moving average costing method. Special materials are valued by DOE.

\section{Property, Plant, and Equipment}

Property, plant, and equipment are purchased, constructed, or fabricated in-house and include major modifications or improvements. These items are capitalized if they have an anticipated service life of two years or more and cost $\$ 50 \mathrm{~K}$ or more. Costs of construction and fabrication are capitalized as construction/fabrication work in process. Upon completion or beneficial occupancy, the value is transferred to the appropriate fixed-assets account. Depreciation is computed using the straightline method over the estimated useful life of the asset.

\section{Liabilities}

Liabilities represent the amount of monies that are likely to be paid by the Laboratory as a result of transactions or events that have already occurred. Liabilities cannot be incurred by LBNL without an authorized appropriation, except for approved unfunded liabilities.

\section{Accrued Annual, Sick, and Other Leave}

Laboratory policy provides for employees' annual vacation benefits ranging from 10 to 16 hours per month. depending upon years of service. Employees may accumulate vacation up to two times their annual leave. Upon retirement or termination, the employee is paid $100 \%$ of accumulated vacation pay.

Each employee accumulates sick leave at a rate of eight hours per month. Unused sick leave accumulates until it is used. If an employee terminates before using sick leave, the benefit is forfeited without liability to the Laboratory. Retiring employees are allowed to apply unused sick leave toward additional years of service.

\section{Retirement Plan}

Most University career employees are participants in the UC Retirement System (UCRS). UCRS consists of a basic 


\section{Summary of Significant Accounting Policies Continued}

defined benefit plan and two voluntary plans composed of several investment funds that are funded with University and employee contributions.

\section{Accounts Receivable}

The following were included in accounts receivable $(\$ K)$ :

\begin{tabular}{|c|c|c|}
\hline & FY2007 & FY2008 \\
\hline Trade Receivables & 910 & 644 \\
\hline Inter-DOE Operations Offices (outside local field office) & 3,164 & 2,086 \\
\hline Intra-DOE Operations Offices (within local field office) & 222 & 354 \\
\hline Employees & 8 & 2 \\
\hline Parent Organization (UC) & $(2,349)$ & $(2,198)$ \\
\hline Reimbursements - Federal Agencies & 8,888 & 6.017 \\
\hline Allowance for Doubtful Accounts & (4) & (4) \\
\hline Total Accounts Receivable - September 30 & 10,839 & 6,901 \\
\hline
\end{tabular}

\section{Inventories}

The following were included in inventories $(\$ K)$ :

\begin{tabular}{|c|c|c|}
\hline & FY2007 & FY2008 \\
\hline Nuclear Materials & 24 & 25 \\
\hline Precious Metals and Other Special Materials & 128 & 115 \\
\hline Stores Inventories & 914 & 377 \\
\hline Allowance for Loss on Stores & $(478)$ & (18) \\
\hline Total Inventories - September 30 & 588 & 499 \\
\hline
\end{tabular}

\section{Other Current Assets}

The following were included in other current assets $(\$ K)$ :

\begin{tabular}{|c|c|c|}
\hline & FY2008 & FY2008 \\
\hline Advances to Other DOE Locations (Russian Subcontracts) & 902 & 301 \\
\hline Prepayments & 265 & 210 \\
\hline Security Deposits & 0 & 5 \\
\hline Total Other Current Assets - September 30 & 1,167 & 516 \\
\hline
\end{tabular}


Note 5

\section{Net Plant and Equipment}

The following were included in net plant and equipment (\$K):

\begin{tabular}{|c|c|c|c|c|c|c|}
\hline \multirow[b]{2}{*}{ Category } & \multicolumn{2}{|c|}{ Plant \& Equip Costs } & \multicolumn{2}{|c|}{$\begin{array}{l}\text { Accumulated } \\
\text { Depreciation }\end{array}$} & \multicolumn{2}{|c|}{ Net Plant \& Equip } \\
\hline & FY2007 & FY2008 & FY2007 & FY2008 & FY2007 & FY2008 \\
\hline Structure, Facilities, \& LI & 385,496 & 393,927 & $(176.718)$ & $(188,107)$ & 208,778 & 205,820 \\
\hline Equipment & 395.722 & 321,335 & $(265,237)$ & $(221,349)$ & 130,485 & 99,986 \\
\hline Assets Under Capital Leases & 25,255 & 72,177 & $(17,221)$ & $(26,031)$ & 8,034 & 46,146 \\
\hline Utilities & 31,786 & 32,151 & $(19,962)$ & $(20,535)$ & 11.824 & 11,616 \\
\hline Reactors \& Accelerators & 140,424 & 144,613 & $(93,382)$ & $(101.743)$ & 47,042 & 42,870 \\
\hline Work in Process & 171,584 & 182,608 & - & - & 171,584 & 182,608 \\
\hline Total & $1,150,267$ & $1,146,811$ & $(572,520)$ & $(557,765)$ & 577,747 & 589,046 \\
\hline
\end{tabular}

Note 6

\section{Drafts Payable}

The following is an analysis of drafts payable (\$K):

\begin{tabular}{|c|c|c|}
\hline & FY2007 & FY2008 \\
\hline Balance - October 1 & 4,656 & 1,017 \\
\hline \multicolumn{3}{|l|}{ Deposits: } \\
\hline Payments Vouchers - Letter of Credit & $(481,141)$ & $(565,199)$ \\
\hline Miscellaneous Receipts & $(43,619)$ & $(48,561)$ \\
\hline Disbursements & 521.120 & 611.767 \\
\hline Drafts Payable Balance - September 30 & 1,017 & (976) \\
\hline
\end{tabular}




\section{Environmental Liability}

The estimated remaining cost of remediation of environmentally contaminated facilities at LBNL is recorded as a liability. The Environmental Management liability is based on baseline life-cycle cost estimates prepared with the DOE Site Office with updates for subsequent changes pursuant to DOE's established change control process.
The Active Facilities liability is based on cost estimates generated for facilities reparted in the Facility Information Management System. The funded portion of the liability is $\$ 1,119 \mathrm{~K}$ and is included in Other Current Liabilities. The following are included in the environmental liability (\$K):

\begin{tabular}{|c|c|c|}
\hline & FY2007 & FY2008 \\
\hline Environmental Management & 9.081 & 9,081 \\
\hline Active Facilities & 769,635 & 603,995 \\
\hline Total Unfunded Environmental Liability - September 30 & 778,716 & 613,076 \\
\hline
\end{tabular}

\section{Capital Leases}

LBNL has entered into capital leases for a Cray Supercomputer, and network equipment. The following is a schedule of future lease payments by fiscal year $(\$ K)$ :

\begin{tabular}{|l|r|}
\hline Fiscal Year & \$K \\
\hline FY2009 & 9,015 \\
\hline FY2010 & 8,820 \\
\hline FY2011 & 1,530 \\
\hline FY2012 & 429 \\
\hline Total Future Payments & $\mathbf{1 9 , 7 9 4}$ \\
\hline
\end{tabular}

\section{Environment, Safety and Health (ES\&H) Liability}

The ES\&H Liabilify is based on ES\&H compliance activities reported annually through the ES\&H Management Plan that are necessary to bring facilities and operations into compliance with existing environmental, safety, and health laws and regulations, excluding activities included in the Environmental Liability. The following are the ES\&H liability (\$K):

\begin{tabular}{|l|r|r|}
\hline & FY2007 & FY2008 \\
\hline Total ES\&H Liability - September 30 & 145,575 & 458,428 \\
\hline
\end{tabular}




\section{Procurement and Property Management}


Table 5.1

\section{Requisitions Submitted by Laboratory Divisions}

\begin{tabular}{|c|c|c|}
\hline Division & \# Requisitions & Estimate ( $\$ \mathrm{~K}$ ) \\
\hline Accelerator \& Fusion Research & 1,931 & $\$ 3,351$ \\
\hline Advanced Light Source & 2,850 & $\$ 4,782$ \\
\hline Chief Financial Officer & 1,437 & $\$ 12,819$ \\
\hline Chemical Sciences & 1.812 & $\$ 3,298$ \\
\hline Computational Research & 445 & $\$ 5,738$ \\
\hline Computing sciences & 985 & $\$ 1,838$ \\
\hline Environmental Energy Technologies & 2,372 & $\$ 8,937$ \\
\hline Engineering & 1,033 & $\$ 2,536$ \\
\hline Environment, Health \& Sofety & 1,422 & $\$ 3,381$ \\
\hline Earth Sciences & 1.984 & $\$ 3,110$ \\
\hline Facilities & 3,322 & $\$ 29,447$ \\
\hline Genomics & 3,679 & $\$ 30,857$ \\
\hline Human Resources & 400 & $\$ 578$ \\
\hline Information Technologies \& Services & 1.465 & $\$ 7.177$ \\
\hline Laboratory Directorate & 443 & $\$ 323$ \\
\hline Life Sciences & 5,926 & $\$ 11.727$ \\
\hline Material Sciences & 7,035 & $\$ 13,553$ \\
\hline NERSC & 219 & $\$ 34.107$ \\
\hline Nuclear Science & 967 & $\$ 2,413$ \\
\hline Operations & 192 & $\$ 139$ \\
\hline Public Affairs & 253 & $\$ 253$ \\
\hline Physical Biosciences & 4,084 & $\$ 41,209$ \\
\hline Physics & 1,124 & $\$ 3,260$ \\
\hline Structural Biology & 60 & $\$ 41$ \\
\hline Technology Services & 11 & $\$ 2$ \\
\hline Institutional Projects & 38 & $\$ 16$ \\
\hline Totals & 45,489 & $\$ 224,892$ \\
\hline
\end{tabular}


Table 5.2

\section{Purchases Placed Using Purchase Orders/Subcontracts}

\begin{tabular}{|l|r|r|}
\hline \multirow{2}{*}{ Total POs } & $(\$ K)$ & \# Actions \\
\cline { 2 - 3 } & $\$ 287,794$ & 31,300 \\
\hline$\$ 0-\$ 2,500$ (non-negative) & $\$ 8,120$ & 26,888 \\
\hline$\$ 2,500-\$ 10,000$ & $\$ 11,081$ & 2,095 \\
\hline$\$ 10,000-\$ 25,000$ & $\$ 16,575$ & 1007 \\
\hline$\$ 25,000-\$ 100,000$ & $\$ 50,679$ & 966 \\
\hline$\$ 100,000-\$ 1,000,000$ & $\$ 78,170$ & 310 \\
\hline$\$ 1,000,000+$ & $\$ 123,169$ & 34 \\
\hline
\end{tabular}

Table 5.3

\section{Purchases Placed Using P-Card}

\begin{tabular}{|l|r|r|}
\hline \multirow{2}{*}{ Total POs } & $(\$ K)$ & \# Actions \\
\cline { 2 - 3 } & $\$ 10,451$ & 15,520 \\
\hline$\$ 0-\$ 500$ & $\$ 1,992$ & 10,239 \\
\hline$\$ 500-\$ 1,000$ & $\$ 1,772$ & 2510 \\
\hline$\$ 1,000-\$ 2,500$ & $\$ 2,962$ & 1909 \\
\hline$\$ 2,500-\$ 5,000$ & $\$ 2,199$ & 630 \\
\hline$\$ 5,000+$ & $\$ 1,526$ & 232 \\
\hline
\end{tabular}

Table 5.4

Laboratory Socioeconomic Performance

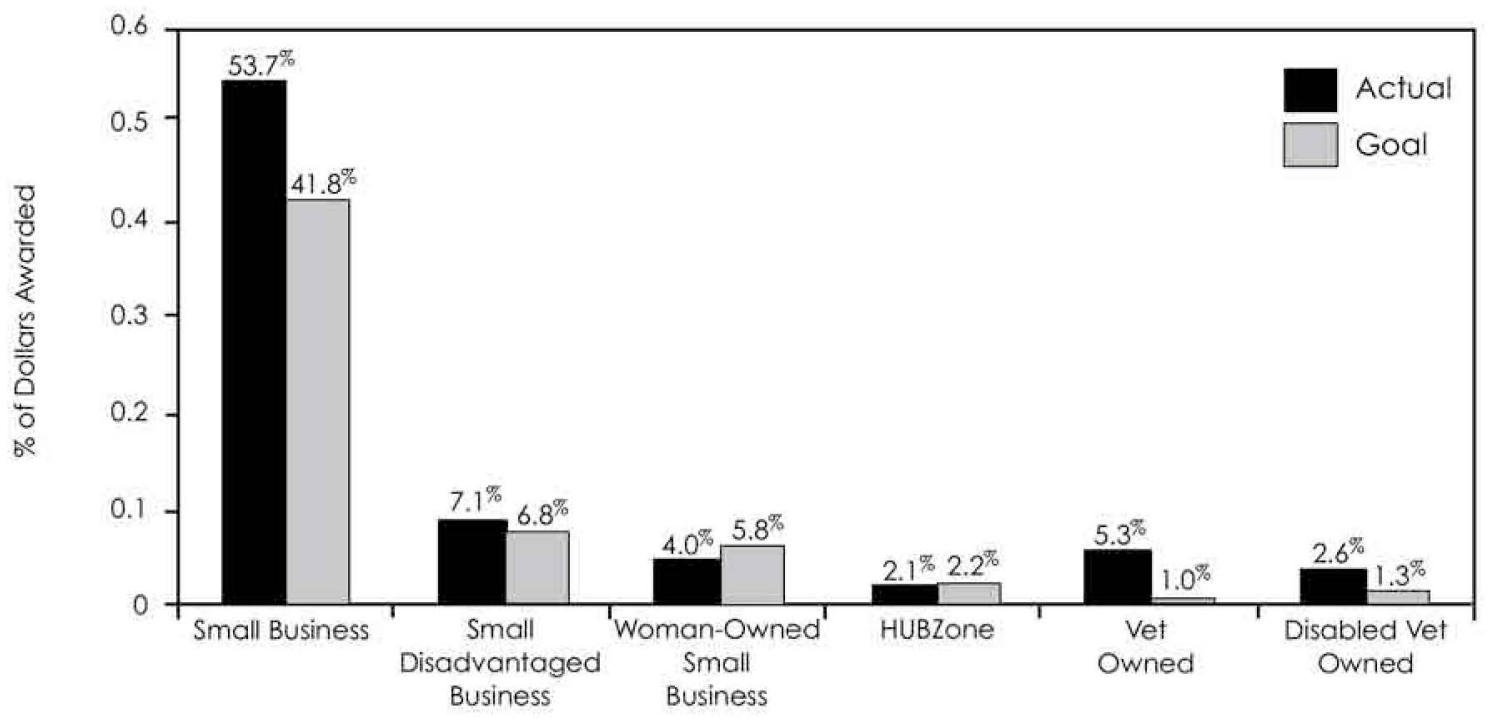


Table 5.5

\section{Property Management Activity}

\begin{tabular}{|c|c|c|c|}
\hline \multicolumn{4}{|c|}{ CHARACTERIZATION OF LABORATORY ASSETS } \\
\hline & \# of Assets & Acquisition Value ( $\$ K$ ) & \\
\hline Total Laboratory Assets & 25,193 & 629,620 & \\
\hline Equipment & 8,875 & 583,057 & \\
\hline Sensitive Assets & 16,318 & 46,563 & \\
\hline Computers Laptops & 2,913 & 6,567 & \\
\hline Computer Desktops & 7,011 & 16,869 & \\
\hline Loaned Assets & 72 & 16,151 & \\
\hline Borrows & 43 & 1.756 & \\
\hline Assets Created in FY2008 & 4,110 & 117,863 & \\
\hline Assets to Excess in FY2008 & 1,517 & 140,322 & \\
\hline Inventory Campaign & Base & Positive Resolutions & \% Positive \\
\hline Controlled & 97 & 97 & 100 \\
\hline Sensitive & 2,603 & 2,596 & 99.7 \\
\hline Validation Size & 53 & 53 & 100 \\
\hline Assets Scanned & 2,700 & 2,524 & 93.5 \\
\hline Division & Quantity & Asset Value (SK) & \\
\hline Accelerator \& Fusion Research & 1.073 & 34.151 & \\
\hline Advanced Light Source & 1,835 & 160.577 & \\
\hline Chief Financial Officer & 339 & 485 & \\
\hline Chemical Sciences & 1,216 & 25,845 & \\
\hline Computational Research & 1,465 & 23,833 & \\
\hline Computing Sciences & 111 & 1.992 & \\
\hline Environmental Energy Technologies & 1,393 & 16,705 & \\
\hline Engineering & 992 & 13,705 & \\
\hline Environment, Health \& Safety & 510 & 3,016 & \\
\hline Earth Sciences & 1,087 & 12,860 & \\
\hline Excess Tum-In Center & 453 & 15,914 & \\
\hline Facilities & 966 & 5,522 & \\
\hline Genomics & 1,794 & 49,086 & \\
\hline Human Relations & 114 & 150 & \\
\hline Information Technologies \& Services & 2,147 & 15,412 & \\
\hline Laboratory Directorate & 162 & 378 & \\
\hline Life Sciences & 1,943 & 29,796 & \\
\hline Material Sciences & 3,082 & 80,699 & \\
\hline NERSC & 1,004 & 72,810 & \\
\hline Nuclear Science & 663 & 28,829 & \\
\hline Operations & 29 & 33 & \\
\hline Public Affairs & 139 & 394 & \\
\hline Physical Biosciences & 1,902 & 23,371 & \\
\hline Physics & 774 & 14,057 & \\
\hline Total & 25,193 & 629,620 & \\
\hline
\end{tabular}




\section{Wr Data from Other DOE Laboratories}


t is sometimes helpful to compare cost/FTE data among national laboratories. However, because the costaccounting systems, overhead definitions, and indirect cost structures can vary greatly between laboratories, benchmarking between organizations is not straight forward. For example, some organizations direct charge activities that others include in overhead. The major idiosyncrasies of each different accounting system are noted in this chapter. Therefore, only general inferences should be drawn from these data. Specific comparisons would be considered invalid.

Table 6.1

\section{Other Laboratories for Which Financial Information is Available}

\begin{tabular}{|l|l|}
\hline Acronym & Laboratory \\
\hline Ames & Ames Laboratory \\
\hline ANL & Argonne National Laboratory \\
\hline BNL & Brookhaven National Laboratory \\
\hline FNAL & Fermi National Accelerator Laboratory \\
\hline LANL & Los Alamos National Laboratory \\
\hline LBNL & Lawrence Berkeley National Laboratory \\
\hline LLNL & Lawrence Livermore National Laboratory \\
\hline ORNL & Qak Ridge National Laboratory \\
\hline PNNL & Pacific Northwest National Laboratory \\
\hline PPPL & Princeton Plasma Physics Laboratory \\
\hline SLAC & SLAC National Accelerator Laboratory \\
\hline SNL & Sandia National Labcratories \\
\hline
\end{tabular}




\section{Summary Cost Data for DOE Laboratories, FY2004 - FY2007 (\$M)}

\begin{tabular}{|c|c|c|c|c|c|c|c|c|c|c|c|c|}
\hline \multirow{2}{*}{ Lab } & \multicolumn{4}{|c|}{ Total Costs } & \multicolumn{4}{|c|}{ Operating Costs } & \multicolumn{4}{|c|}{ FTEs } \\
\hline & FY2004 & FY2005 & FY2006 & FY2007 & FY2004 & FY2005 & FY2006 & FY2007 & FY2004 & FY2005 & FY2006 & FY2007 \\
\hline Ames & 29.5 & 30.5 & 33.2 & 29.4 & 26.4 & 27.1 & 27.2 & 27.2 & 318 & 320 & 313 & 306 \\
\hline ANL (a) & 569.7 & 520.7 & 512.3 & 534.2 & 520.9 & 482.1 & 474.0 & 493.5 & 3,789 & 2,994 & 2,893 & 2,830 \\
\hline BNL & 454.4 & 465.1 & 472.5 & 495.6 & 413.6 & 428.6 & 405.3 & 430.2 & 2,700 & 2,617 & 2,510 & 2,540 \\
\hline FNAL (a) & 317.0 & 317.9 & 328.1 & 337.2 & 259.3 & 267.5 & 284.1 & 322.0 & 2.011 & $n / p$ & 1,945 & 1,977 \\
\hline LANL & $1,996.2$ & $2,101.2$ & $2,145.2$ & $2,046.3$ & $1,798.1$ & $1,841.7$ & 1.906 .9 & $1,815.5$ & 8,591 & 8,992 & 9,081 & 8,764 \\
\hline LBNL (a) & 504.0 & 523.8 & 517.2 & 516.4 & 435.8 & 433.0 & 452.9 & 465.9 & 2,982 & 2,891 & 2,766 & 2,723 \\
\hline LLNL (a) & $1,629.7$ & $1,625.8$ & $1,600.7$ & 1.623 .6 & $1,452.6$ & $1,483.0$ & $1,451.8$ & $1,496.0$ & 7.713 & 7,661 & 7,421 & 7,191 \\
\hline ORNL & $1,025.7$ & $1,025.7$ & 989.3 & $1,100.0$ & 751.4 & 863.7 & 889.5 & $1,013.0$ & 3,930 & 4,035 & 4,137 & 4,238 \\
\hline $\begin{array}{l}\text { PNNL } \\
(a, b)\end{array}$ & 558.7 & 648.8 & 678.6 & - & 545.9 & 634.0 & 662,1 & - & 3,006 & 3,224 & 3,326 & - \\
\hline PPPL (a) & 75.2 & 81.8 & 79.5 & 77.4 & 56.7 & 58.1 & 54.0 & 53.9 & 470 & 455 & 448 & 457 \\
\hline SLAC & 255.1 & 292.6 & 355.9 & 360.4 & 207.5 & 205.0 & 214.9 & 233.0 & 1,645 & 1,606 & 1,616 & 1,662 \\
\hline SNL (a) & $2,227.0$ & $2,302.4$ & $2,302.9$ & $2,298.6$ & $1,941.2$ & $2,059.0$ & $2,077.2$ & $2,155.7$ & 8,294 & 8,561 & 8,625 & 8,478 \\
\hline \multicolumn{13}{|c|}{ (a) Operating costs exclude EQU and GPP. } \\
\hline \multicolumn{13}{|c|}{ (b) PNNL did not submit FY2007 data. } \\
\hline
\end{tabular}




\section{Overhead Information for DOE Laboratories, FY2007}

\begin{tabular}{|c|c|c|c|c|c|c|}
\hline Lab & $\begin{array}{l}\text { Overhead Costs } \\
\qquad(\$ M)\end{array}$ & $\begin{array}{l}\text { Distribution Base } \\
\qquad(\$ M)\end{array}$ & $\begin{array}{c}\text { Overhead Rate as Applied to } \\
\text { Distributed Base (\%) }\end{array}$ & $\begin{array}{l}\text { Operating } \\
\text { Costs (SM) }\end{array}$ & $\begin{array}{c}\text { Overhead As a \% } \\
\text { of Operating }\end{array}$ & \\
\hline Ames & 10.0 & 19.4 & $51.5(\mathrm{a})$ & 27.2 & 35.3 & (b) \\
\hline ANL & 108.9 & 422.7 & (c) & 493.5 & 22.1 & \\
\hline BNL & 92.5 (d) & $262.6 / 253.1(\mathrm{e})$ & $8.25 / 26.3(f)$ & 430.2 & 21.5 & \\
\hline FNAL & 69.9 & 267.3 & 26.2 & 322 & 21.7 & \\
\hline LANL & 385.0 & (g) & (g) & $1,815.5$ & 21.0 & \\
\hline LBNL & $97.6(\mathrm{~h})$ & 202.5 (i) & 48.2 & 465.9 & 20.9 & \\
\hline LLNL & 276.3 (j) & 756.9 & 36.5 & $1,496.0$ & 18.5 & \\
\hline ORNL & 217.9 & $480.7(k)$ & 45.3 & $1,013.0$ & 21.5 & \\
\hline PNNL & - & - & $=$ & - & - & (o) \\
\hline PPPL & 25.4 & (I) & (1) & 53.9 & 35.2 & $(m)$ \\
\hline SLAC & 60.2 & 296.9 & 20.3 & 233.0 & 25.8 & \\
\hline SNL & 311.3 & (n) & $|n\rangle$ & 2.155 .7 & 14.4 & \\
\hline
\end{tabular}

a. Ames-Overhead is comprised of three pools: Site at $56 \%$. Procurement at $18 \%$, and G\&A at $11.3 \%$.

b. Ames-Excludes overhead costs distributed to capital funds. ( $\$ 0.4 \mathrm{M}$ in FY2007)

c. ANL-The various rates in FY2007 are: Materials/Subcontracts 6.7\%, Service Centers 17.5\%, Common Support 25.1\%, LDRD 7.2\%, G\&A 3.0\%.

d. BNL-Includes Common Support and Traditional G\&A only. Costs for LDRD, material burden, and space recharge pools are not part of these costs.

e. BNL-Distribution base represents the Traditional/Common Support base for the standard G\&A rates. Taxable base for special rates not included. Beginning in FY2005, BNL includes overhead costs distributed to operating-funded accounts only.

f. BNL-The following are the standard G\&A rates applied to the majority of projects: $8.25 \%$ is the traditional G\&A rate applied on total modified costs plus R\&D subcontracts and special pracurements less central recharges and central allocations: $31.2 \%$ is the common support G\&A rate applied on total modifled costs only. Total G\&A rate is $39,45 \%$.

g. LANL-Distribution base and overhead rate are not available as a single value because of multiple allocation bases.

h. LBNL-Includes overhead costs distributed to operating-funded accounts only.

i. LBNL-G\&A is distributed over a value-added base, consisting of total costs less direct materials and subcontracts. Distribution base represents the normalized standard base.

j. LLNL-G\&A is distributed over a value-added base, consisting of total costs less direct materials and subcontracts. Distribution base represents the normalized standard base.

k. ORNL-Uses different distribution bases for each overhead pool. The data shown here represent the G\&A base, which is distributed over a tatal modified cost base.

I. PPPL-Includes all overhead costs except LDRD. Distribution base and overhead rate are not available as a single value due to multiple pools and allocation bases.

$m$. PPPL-Excludes $\$ 6.5 \mathrm{M}$ of overhead costs distributed to capital funds.

n. SNL-G\&A distribution base is modified total cost base. SNL distribution base and overhead rate are not available as a single value because of multiple allocation bases.

o. PNNL did not submit $\mathrm{FY}_{2007}$ data. 
Table 6.4

Overhead Costs as a Percentage of Operating Costs for DOE Laboratories, FY2004 - FY2007

\begin{tabular}{|c|c|c|c|c|c|c|c|c|}
\hline \multirow{2}{*}{\begin{tabular}{|l} 
Laboratory \\
Ames \\
\end{tabular}} & \multicolumn{2}{|l|}{ FY2004 } & \multicolumn{2}{|c|}{ FY2005 } & \multicolumn{2}{|l|}{ FY2006 } & \multicolumn{2}{|l|}{ FY2007 } \\
\hline & 31.1 & (a) & 32.5 & (a) & 34.2 & (a) & 35.3 & (a) \\
\hline ANL & 19.9 & & 19.1 & & 20.5 & & 22.1 & \\
\hline BNL & 21.6 & & 21.3 & & 21.4 & & 21.5 & \\
\hline FNAL & 22.4 & (b) & $n / p$ & & 21.3 & (b) & 21.7 & (b) \\
\hline LANL & 20.4 & & 19.5 & & 19.9 & & 21.2 & \\
\hline LBNL & 21.6 & (c) & 22.1 & (c) & 20.0 & (c) & 20.9 & (c) \\
\hline LLNL & 18.9 & & 19.1 & & 18.4 & (d) & 18.5 & \\
\hline ORNL & 21.3 & & 19.9 & & 23.1 & & 21.5 & \\
\hline PNNL & 18.6 & & 16.5 & & 18.6 & & $=$ & (f) \\
\hline PPPL & 34.6 & (e) & 33.7 & (e) & 34.8 & (e) & 35.2 & (e) \\
\hline SLAC & 22.9 & & 24.2 & & 26.0 & & 25.8 & \\
\hline SNL & 14.7 & & 14.9 & & 15.4 & & 14.4 & \\
\hline \multicolumn{9}{|c|}{$\begin{array}{l}\text { (a) Ames excludes overhead costs distributed to capital funds. ( } \$ 0.4 \mathrm{M} \text { in FY2007) } \\
\text { (b) FNAL excludes overhead costs distributed to capital funds. (\$3.3M in FY 2007) } \\
\text { (c) LBNL includes overhead costs distributed to operating funded accounts only. } \\
\text { (d) LLNL restructured overhead costs in FY2006; it includes G\&A and SMS (Strategic Mission Support) costs, but excludes } \\
\$ 1.2 \mathrm{M} \text { of overhead costs distributed to DOE capital accounts. } \\
\text { (e) PPPL excludes overhead costs distributed to capital funds. ( } \$ 6.5 \mathrm{M} \text { in FY2007). } \\
\text { (f) PNNL did not submit FY2007 data. }\end{array}$} \\
\hline
\end{tabular}

Figure 6.1

\section{Functional Support Costs as a Percent of Total Costs, FY2002 - FY2007}

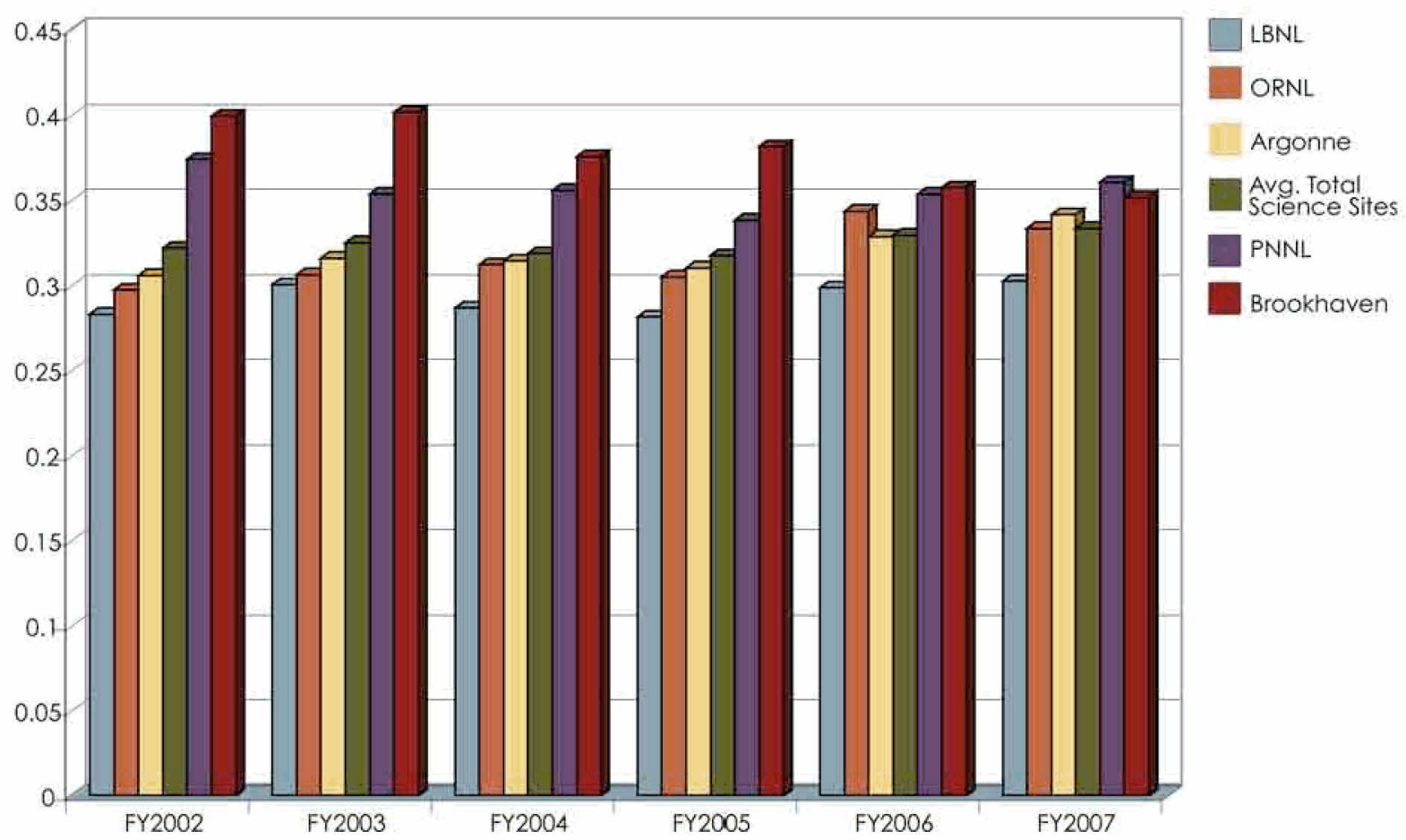


$\Downarrow$ 7. Acronyms and Key Terms

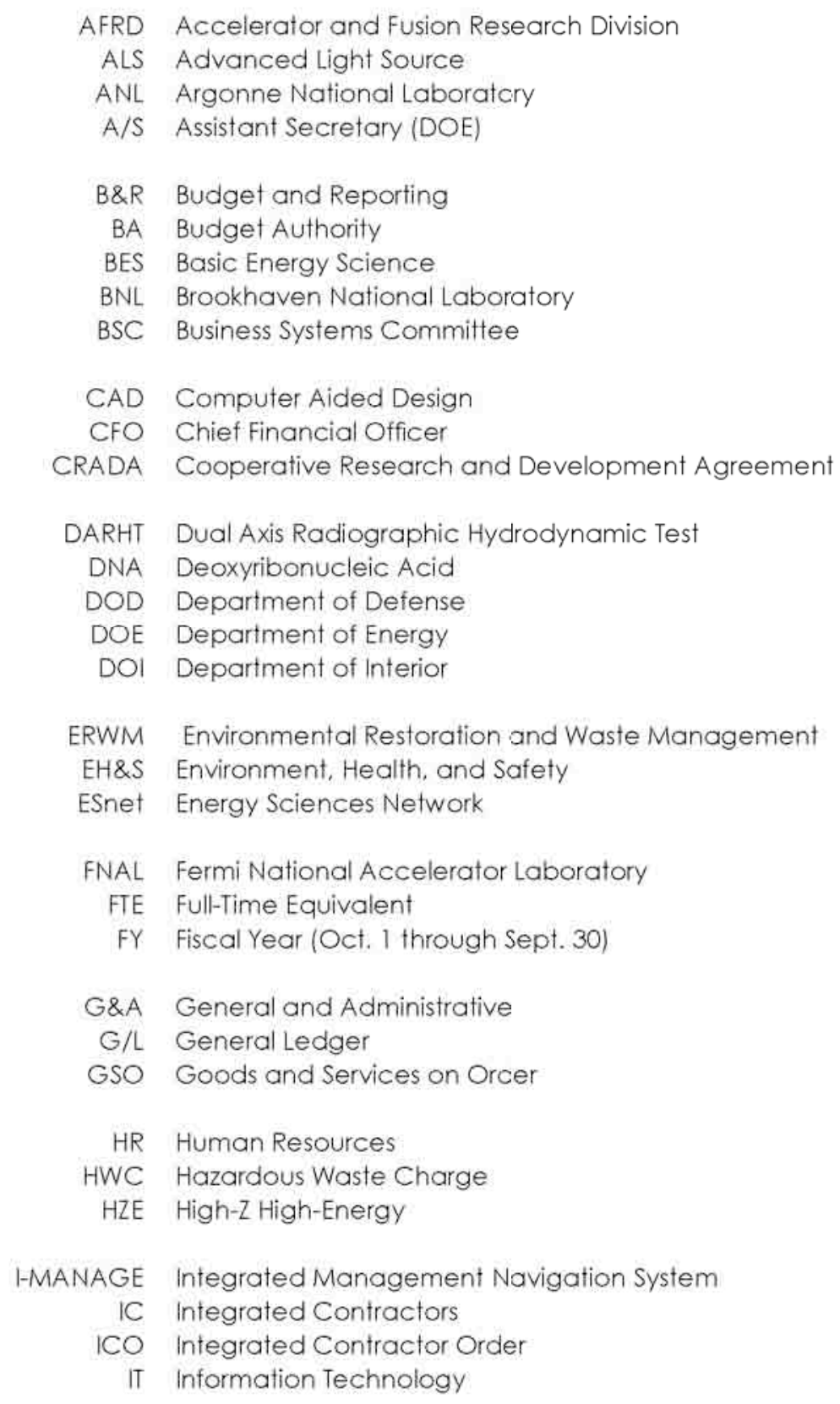




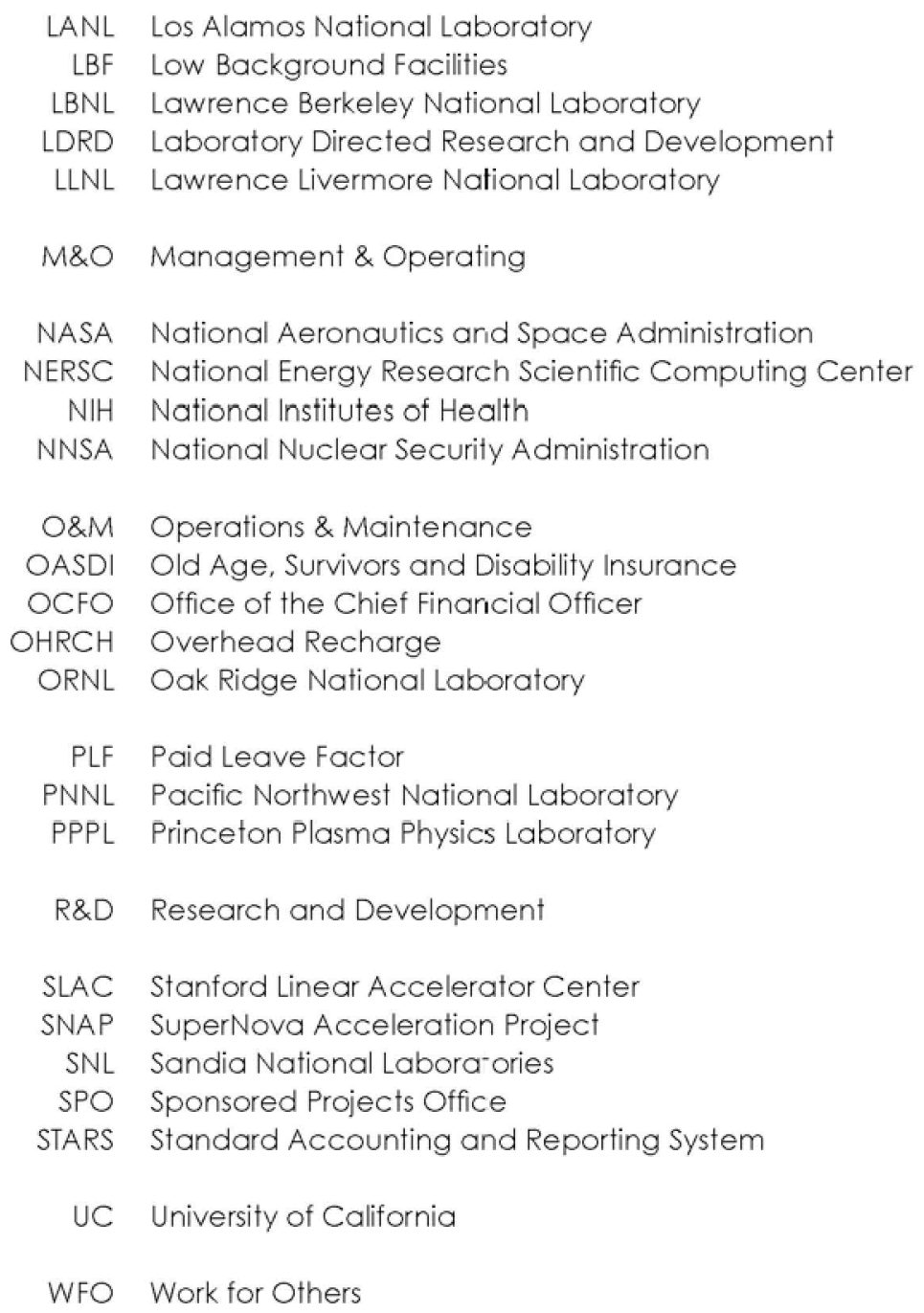

\section{Key Terms}

Throughout this document, \$K means dollars in thousands, \$M means dollars in millions, and \$B means dollars in billions. 
This document was prepared as an account of work sponsored by the United States Government. While this document is belleved to contain correct information, neither the United States Government nor any agency thereof, nor the Regents of the University of California, nor any of thell employees, makes any warranty, express or implied, or assumes any legal responsibility for the accuracy, completeness, or usefulness of any information, apperatus, product, or process disclosed, or represents that its use would not infringe privately owned rights. Reference herein to any specific commercial product, process, or service by its trade name, trademark, manufacturer, or otherwise, does not necessarIly constitute or imply its endersement, recommendation, or fovering by the lunited states covernment or any agency thereof, or the Regents of the University of Californild. The views and opinions of authors expressed herein do not necessarily state or reflect those of the United States Government or any agency thereof or the Regents of the University of California. 